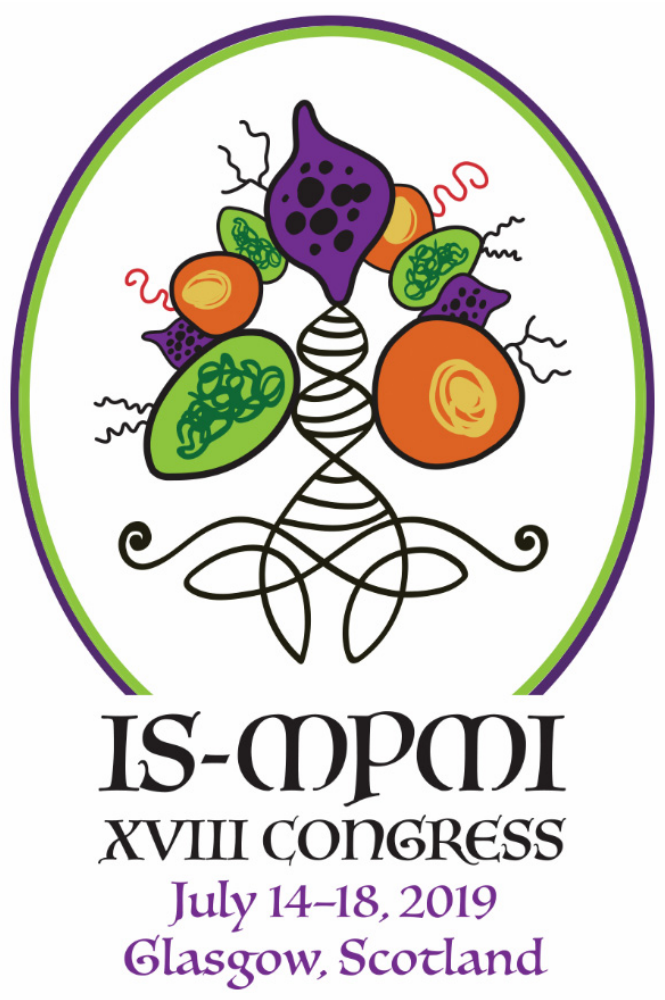

\title{
IS-MPMI XVIII Congress Abstracts of Concurrent Session Presentations
}

\begin{abstract}
IS-MPMI XVIII Congress in Glasgow, Scotland, July 14-18, 2019. The recommended format for citing congress abstracts, using the first abstract below as an example, is as follows:

Manley, B., Sawers, R., Riahy, D., Li, B., and Paszkowski, U. 2019. Independent of arbuscular mycorrhizal symbiosis: Positional cloning and characterisation of a novel arbuscular mycorrhizal mutant in Zea mays. (Abstr.) Molecular Plant-Microbe Interactions 32:S1.213. https://doi.org/10.1094/MPMI-32-10-S1.213
\end{abstract}

The abstracts are published as a supplement to $M P M I$ for citation purposes. They were not reviewed by the $M P M I$ Editorial Board and were not edited by the APS editorial staff.

https://doi.org/10.1094/MPMI-32-10-S1.213

(C) 2019 International Society for Molecular Plant-Microbe Interactions

Independent of Arbuscular Mycorrhizal Symbiosis: Positional cloning and characterisation of a novel arbuscular mycorrhizal mutant in Zea mays

B. MANLEY (1), R. Sawers (2), D. Riahy (1), B. Li (3), U. Paszkowski (4), (1) University of Cambridge, Cambridge, U.K.; (2) Department of Plant Science, Penn State, U.S.A.; (3) Corteva Agriscience, IA, U.S.A.; (4) University of Cambridge, U.K.

Arbuscular mycorrhizal fungi from the Glomeromycota phylum form a beneficial symbiotic relationship with the root systems of a majority of plant species, enabling plants to profit from increased phosphate uptake efficiency. Further understanding of this interaction may prove vital in meeting the challenge of an increasing demand for phosphate supply in agriculture. Establishment of this symbiotic interaction relies on molecular crosstalk between the fungus and the plant roots in the rhizosphere, even before physical contact has occurred. A forward genetic screen has identified independent of arbuscular mycorrhizal symbiosis (ina), a Zea mays mutant phenotype that displays a complete block in colonisation when inoculated with Rhizophagus irregularis. Positional cloning methods have revealed a candidate gene behind this phenotype, and extensive phenotyping has established that this maize mutant lacks the ability to signal to its symbiotic partner, abolishing the ability to promote their beneficial relationship. To identify the impact of the removal of this signal, a transcriptomics analysis of Rhizophagus irregularis spores during their early interaction with this Zea mays mutant has been carried out to further examine the extended phenotype of the loss of this plant gene. 
Convergent gene loss in aquatic plants reveals novel plant immunity and drought responses components

E. L. BAGGS (1), R. O’Grady (2), C. Schudoma (3), A. Thanki (3), W. Haerty (3), K. V. Krasileva (4), (1) UC Berkeley, Berkeley, CA, U.S.A.;

(2) The Sainsbury Laboratory, U.K.; (3) Earlham Institute, U.K.; (4) University of California Berkeley, Berkeley, CA, U.S.A.

Plants colonization of land from water altered selection on numerous traits. A high dependence on microbes was crucial during this transition. Since, there have been several migrations within monocots and dicots to an aquatic environment however, little is known about the effect of such a change on plant-microbe interactions. We annotated the immune receptor family, Nucleotide Binding Leucine Rich Repeat proteins (NLRs), across 19 flowering plants genomes and identified four aquatic species with low numbers of NLR-encoding genes relative to other plants. The loss of NLRs in these aquatic species is not random with no remaining TIR-1 and RPW8 NLRs. Most importantly all three members of the EDS1, PAD4 and SAG101 signaling complex are absent in all four species. These observations indicate a convergent loss of this signaling pathway among the four species representing two independent transitions to water. We identified 44 additional genes lost only in aquatic species, which we named AngioSperm Terrestrial-Retained, Aquatic-Lost (ASTRAL). We hypothesised that ASTRAL genes may be involved in defense response similarly to the lost NLRs and EDS1 complex. Fifteen $A S T R A L$ genes are differentially expressed upon pathogen infection. Whilst $11 A S T R A L$ genes were differentially expressed upon drought. The $A S T R A L$ gene set provides information on pathways required for adaptation to land and novel candidates for roles in plant immunity functions previously obscured by genetic redundancy.

Developing CRISPR-Cas13 for multi-use RNA-targeting in plants

D. E. COOK, V. Sharma, W. Zheng, J. Huang, W. Zhang, Kansas State University, Manhattan, KS, U.S.A.

New technological advances are needed to meet $21^{\text {st }}$ century agricultural demands. The development of the clustered regularly interspaced short palindromic repeat (CRISPR) and associated proteins (Cas) for DNA editing holds significant promise to help increase agricultural productivity. However, targeting and editing DNA has limitations for multigene traits and cannot provide RNA-virus resistance. As an alternative technology, we have developed the programmable RNA-guided RNA-targeting type VI CRISPR-Cas system, termed Cas13, for use in plants. There are few reports regarding Cas 13 function in plants and further molecular characterization is needed. We developed two Cas 13a systems from Leptotrichia buccalis (Lbu) and Lachnospiraceae bacterium (Lba) as a platform for targeting trans, endogenous and viral RNA. The function of Cas13 was tested in Nicotiana benthamiana and Arabidopsis thaliana using biochemical and genomic techniques and the results show that both tested Cas13s can significantly reduce diverse target mRNAs. Unexpectedly, expressing a Cas13 CRISPR-RNA designed to express multiple guides provided target mRNA reduction independent of the Cas13 protein. Stable transgenic Arabidopsis plants have been developed to further understand this phenomenon. Collectively, this research provides molecular characterization of two Cas 13a systems in plants to aid in new approaches for plant biotechnology, anti-viral resistance and functional genomics.

A genomic map of local adaptation to microbiota and potential pathobiota in Arabidopsis thaliana C. Bartoli (1), L. Frachon (2), F. ROUX (3), (1) IGEPP, Université de Rennes, France; (2) DSEB, University of Zurich, Switzerland; (3) LIPM, Université de Toulouse, INRA, CNRS, INPT, Castanet-Tolosan, France

In natura, hosts are often infected by multiple pathogens. Therefore, current challenges in microbial pathogenesis are (i) to explore the relationships between whole microbial communities inhabiting hosts (microbiota) and microbes with potential pathogenic behavior (pathobiota) in a realistic ecological context, and (ii) to fine map host genes controlling the whole pathobiota. Here, we investigated the in situ bacterial microbiota-pathobiota relationships in 163 ecologically contrasted Arabidopsis thaliana populations located in the south-west of France. In agreement with the theory of the invasion paradox, we observed a highly significant hump-backed curve between pathobiota and microbiota $\alpha$ diversity. In most populations, we also observed a strong dynamics of microbiota composition between seasons. Accordingly, the pathobiota composition was explained by combinations of season-specific microbiota OTUs. Finally, we conducted a Genome Environment Association using more than 1.5 million SNPs analysis to finely map genomic regions in A. thaliana associated with bacterial community descriptors. We mapped adaptive QTLs associated with the presence/absence of OTUs as well as for diversity and composition of communities, thereby highlighted the benefit of exploring diffuse biotic interactions. This study provides a first step to study the ecological genomics of coevolutionary processes between $A$. thaliana and its bacterial social network I south-west of France.

\section{Soil-borne legacies of plant disease}

R. L. BERENDSEN, G. Vismans, Y. Song, P. A. H. M. Bakker, C. M. J. Pieterse, Plant-Microbe Interactions, Department of Biology, Utrecht University, Utrecht, Netherlands

Plants can adjust their root microbiome upon pathogen infection and specifically recruit resistance-inducing microbes. As a result, infected plants can create a soil-borne legacy of disease that confers enhanced protection against the pathogen in a second population of plants growing in the same soil. We want to shed light on the genetic mechanism by which plants recruit beneficial microbes in response to attack and find out whether plants respond differently to pathogens with distinct lifestyles. To this end, we analyzed root transcriptomic responses of Arabidopsis plants inoculated with distinct leave pathogens to identify genes or processes that are involved in the creation of soil-borne legacies. Moreover, the rootspecific transcription factor MYB72 previously emerged as an important regulator of rhizobacteria-induced systemic resistance (ISR). MYB72 is required for the systemic onset of ISR and it regulates the biosynthesis of coumarins that affect the composition of the root microbiome. We found that mutant $m y b 72$ plants are still able to perceive a soil-borne legacy created by wild-type downy-mildew-infected plants. However, myb72 plants infected by downy mildew did not improve the resistance of wild-type plants subsequently growing on the same soil. This indicates that MYB72, and likely coumarins, are required for the creation of soil-borne legacies of plant disease. We are currently exploring the role of coumarins in the creation of resistance-inducing microbiomes. 
Structural and Biochemical Analysis of Plant TIR NAD+ Cleavage Activity

H. BURDETT (1), M. X. Rank (1), J. Chen (2), X. Zhang (3), Y. Shi (4), T. Ve (4), P. N. Dodds (5), B. Kobe (1), (1) University of Queensland, Brisbane, QLD, Australia; (2) Commonwealth Scientific and Industrial Research Organisation, Canberra, ACT, Australia; (3) The Australian National University, Canberra, ACT, Australia; (4) Griffith University, Southport, QLD, Australia; (5) CSIRO Agriculture and Food, Canberra, ACT, Australia

Plant NLRs (Nucleotide binding, Leucine-rich repeat Receptors) provide resistance to a range of biotrophic pathogens, by recognition of avirulence factors, and proceeded by initiation of downstream immune responses. NLR signaling is transduced through the N-terminal TIR domain, and resistance is often characterized by localized cell death around the site of infection. How plant TIR domains transduce this signal is still unknown, however, like TIR domains from mammalian NLRs, plant TIR domains form homo- and heterotypic dimers, and this dimerization is required for signaling. Recently, a TIR domain from the mammalian TLR adaptor family, SARM1, was shown to possess NADase activity, the first report of a TIR domain displaying enzymatic activity. Given the structural similarities between SARM TIR and plant TIR domains, the requirement of SARM dimerization for NADase activity, and the lack of downstream binding partners identified for plant NLRs, we hypothesize that some plant TIR domains also possess NADase activity. Here we present biochemical and structural evidence that plant TIR domains possess NADase activity, and new insights into how this activity is involved in eliciting HR. NAD+ is cleaved into ADPR and Nicotinamide in vitro by the TIR domains from RUN1 and L6. NADase activity can be increased by addition of macromolecular crowding agents. Mutations to the NADP+ binding site in the RUN1 structure also effect NADase activity.

Conserved Biochemical Defenses Underpin Host Responses to Oomycete Infection in an Early Divergent Land Plant Lineage P. CARELlA (1), A. Gogleva (1), D. J. Hoey (1), A. Bridgen (1), S. C. Stolze (2), H. Nakagami (3), S. Schornack (1), (1) Sainsbury Laboratory, University of Cambridge, Cambridge, U.K.; (2) Max Planck Institute for Plant Breeding Research, Cologne, Germany; (3) Max Planck Institute for Plant Breeding Research, Germany

The expansion of plants onto land necessitated the evolution of robust defense strategies to protect against a wide array of microbial invaders. While host responses to microbial colonization are extensively explored in evolutionarily young land plant lineages like angiosperms, we know relatively little about plant-pathogen interactions in earlier diverging land plants thought to better represent the ancestral state. Here, we define the transcriptional and proteomic response of the early divergent liverwort Marchantia polymorpha to infection with the oomycete pathogen Phytophthora palmivora. We uncover a robust molecular response to oomycete colonization in Marchantia that consists of conserved land plant genes. Direct macroevolutionary comparisons of host infection responses in Marchantia and Nicotiana benthamiana revealed a shared set of orthologous microbe-responsive genes involved in phenylpropanoid metabolism. In addition, we identify a role for the R2R3-Myb transcription factor MpMyb14 in activating phenylpropanoid (flavonoid) biosynthesis during oomycete infection. Ectopic overexpression of MpMyb14 led to the accumulation of anthocyanin-like pigments and dramatically enhanced liverwort resistance to $P$. palmivora infection. Collectively, our results demonstrate that the Marchantia response to oomycete infection displays evolutionarily conserved features indicative of an ancestral pathogen deterrence strategy centered on phenylpropanoid-mediated biochemical defenses.

Novel alpha-SNAP and NSF mechanisms mediate disease resistance against cyst nematodes A. F. BENT (1), A. Bayless (2), K. Butler (3), R. Zapotocny (2), J. Smith (2), C. Fliege (4), M. Hudson (4), B. Diers (4), S. Chen (5), X. Wang (6), D. Grunwald (2), S. Han (2), (1) University of Wisconsin-Madison, Madison, WI, U.S.A.; (2) University of Wisconsin - Madison, Madison, WI, U.S.A.; (3) Anderson University, Anderson, IN, U.S.A.; (4) University of Illinois at Urbana-Champaign, Urbana, IL, U.S.A.; (5) Cornell University, Ithaca, NY, U.S.A.; (6) USDA-ARS, Ithaca, NY, U.S.A.

Soybean cyst nematode (SCN) is the most yield-reducing pathogen of U.S. soybeans. We will describe new discoveries about the mechanisms of soybean resistance to SCN. Soybean breeders rely heavily on the complex Rhgl locus. We previously reported that one of the Rhgl genes encodes an atypical, cytotoxic $\alpha$-SNAP protein that disrupts host cell vesicle trafficking. $\alpha$-SNAP and NSF are essential eU.K.aryotic housekeeping proteins that normally interact to sustain vesicular trafficking. We have now learned that $100 \%$ of soybean lines with Rhgl carry, at an unlinked locus, the gene for an NSF protein with unusual amino acids at the predicted $\alpha$-SNAP/NSF interaction site. This NSF-RAN07 exhibits better interaction with Rhgl $\alpha$-SNAPs and partially mitigates their cytotoxicity. Separately, we learned that Rhgl-mediated resistance can involve depletion of wild-type $\alpha$-SNAP abundance. In unpublished work, we have found that the responsible gene at another SCN resistance QTL encodes a gamma-SNAP protein. Hence for disease resistance, SCN selection pressure has apparently driven multiple types of re-wiring of soybean NSF and SNAP proteins, which in other organisms are highly conserved components of the SNARE recycling machinery. Transgenic transfer of this cyst nematode resistance can function in other plant species against other cyst nematodes. Lastly, new findings reveal that Rhgl is a resistance pyramid with different products expressed at different stages of the infection process.

Molecular mechanisms of AvrPiz-t-Piz-t-mediated immunity against Magnaporthe oryzae Y. NING (1), X. Shi (1), R. Wang (1), C. H. Park (2), G. L. Wang (2), (1) Institute of Plant Protection, Chinese Academy of Agricultural Sciences, China; (2) Department of Plant Pathology, The Ohio State University, Columbus, OH, U.S.A.

Rice blast, caused by the fungal pathogen Magnaporthe oryzae, is one of the most serious rice diseases. We previously cloned the broad-spectrum resistance (R) gene Piz- $t$ in rice and its cognate Avr gene AvrPiz-t in M. oryzae. To elucidate the molecular mechanism underlying AvrPiz-t-Piz-tmediated rice immunity against $M$. oryzae, we performed a yeast two hybrid screen to identify potential host targets of AvrPiz-t in rice. Among the twelve APIPs (AvrPiz-t Interacting Proteins), APIP5 is a bZIP-type transcription factor, APIP6 is a RING-type E3 ligase, and APIP7 is the potassium channel OsAKT1 protein. Using genetic and molecular methods, we demonstrated that AvrPiz-t targets APIP5, APIP6 and OsAKT1 for suppression of the PAMP-triggered immunity (PTI) in rice. Mechanistically, AvrPiz-t specifically suppresses APIP5 transcriptional activity and OsAKT1-mediated $\mathrm{K}^{+}$currents. APIP6 is a positive regulator of PTI and ubiquitinates and degrades OsELF3-2 in rice that plays a negative role in rice immunity to M. oryzae. We also found that APIP5 positively regulates the accumulation of Piz-t. Interestingly, Piz-t can stabilize APIP5 to prevent necrosis at the necrotrophic stage. Taken together, these results indicate that the fungal effector AvrPiz-t targets multiple rice proteins that play important roles in both PTI and effector-triggered immunity. 
Evolution of the nitrogen-fixing root nodule symbiosis

M. PARNISKE, Genetics, Faculty of Biology, University of Munich (LMU), Biocenter Martinsried, Martinsried, Germany

The nitrogen-fixing root nodule symbiosis is phylogenetically restricted to a single clade of plants comprising four orders, the Fabales, Fagales Cucurbitales and Rosales $(\mathrm{FaFaCuRo})$. Its restricted and yet scattered occurrence within that clade has led to the hypothesis that its common ancestor has acquired a predisposition that subsequently enabled multiple independent evolutionary inventions of nodulation. However, the molecular and biological nature of the predisposition event has remained enigmatic. Intracellular bacteria are - with the only exception of Gunnera- exclusively found within the FaFaCuRo clade, which led to the hypothesis that the predisposition event enabled the common ancestor to take up bacteria into living plant cells. We compared plant genomes of key species previously described to identify genetic elements and molecular mechanisms in the evolution of the nitrogen-fixing root-nodule symbiosis. We analyzed the relevance of the identified elements in planta to unravel the possible evolutionary role of such acquisitions. Our results revealed a key step in the evolution of the nitrogen-fixing rootnodule symbiosis.

Stealth pathogen: Tomato immune suppression by effector modification.

J. JELENSKA, J. Lee, A. J. Manning, D. Wolfgeher, Y. Ahn, G. Walters-Marrah, L. Garcia, I. Lopez, S. J. Kron, J. T. Greenberg, University of Chicago, Chicago, IL, U.S.A.

The plant pathogen Pseudomonas syringae secretes multiple effectors to suppress plant defenses. Some effectors are recognized by plants and others may suppress the resulting immune responses. HopZ3 of Psy B728a is an aminotransferase targeting multiple components of plant immune complexes, but also Psy effectors activating these complexes. In Arabidopsis, HopZ3 acetylates the host RPM1 complex and Psy AvrRpm1 and AvrB3 effectors. In $P$. syringae resistant tomato, the main immune complex includes PRF and PTO kinase. We found that HopZ3 acts as a virulence factor on tomato by suppressing defenses triggered by Psy AvrPto1. HopZ3 interacts with members of PTO complex and acetylates PTO, AvrPto1, SIRIPK and SIRIN4s in vitro and in planta. Mapping HopZ3-dependent acetylation sites in planta revealed that AvrPto1 is modified on histidine, serine and threonine residues, some of which affect binding to PTO. In PTO, HopZ3 acetylates the activation loop important for kinase activity and recognition of AvrPto1. We found that acetylation of either AvrPto1 or PTO by HopZ3 reduces their binding in vitro. Mutation of acetylation sites in AvrPto1 decreases its recognition in tomato in the absence of HopZ3 and increases bacterial growth. Moreover, acetylation of SIRIPK by HopZ3 reduces its activity as well as phosphorylation of HopZ3 and SIRIN4s. Psy modulation of both its own avirulence effectors and their plant targets leads to decreased defenses and increased susceptibility to infection.

Signaling and Nutrient in Plant-Microbe Sybmioses

E. WANG, Institute of Plant Physiology and Ecology, CAS, Shanghai, China

Most land plant species enter a mutualistic symbiosis with arbuscular mycorrhizal (AM) fungi to improve nutrient acquisition from the soil. In return, up to $20 \%$ of host plant photosynthate is transferred to the mycorrhizal fungus in the form of lipids and sugar. Nutrient exchange must be regulated by both partners in order to maintain a stable symbiotic relationship. However, the mechanisms that regulate lipid transfer from plant to AM fungus are elusive. Here, we show that MtAP2-domain transcription factors are master regulators of AM symbiosis by controlling lipid transfer and periarbuscular membrane formation. For instance, MtWRI5 binds to AW-box cis-regulatory elements in promoters of the lipid transporter STR and phosphate transporter MtPT4 in M. truncatula. Mtwri5 mutants displayed impaired arbuscule formation, whereas overexpression of MtWRI5 activates FatM, STR and MtPT4, suggesting that MtWRI5 controls bidirectional nutrient exchange in AM symbiosis. Finally, we showed that MtWRI5 and RAMI regulate each other at the transcriptional level, forming a positive loop to orchestrate bidirectional nutrient transfer between two symbionts. Our data suggest a role for MtWRIS in controlling lipid transfer and periarbuscular membrane formation via regulation of fatty acid biosynthesis and transporter genes in arbuscule-containing cells. I will also discuss our new finding on recognition of mycorrhizal fungus.

Isolation and analysis of RNA cargo in extracellular vesicles of Ustilago maydis

S. KWON (1), A. Brachmann (2), M. Feldbrügge (3), (1) Heinrich-Heine-Universität Düsseldorf, Düsseldorf, Germany; (2) Biozentrum der LMU Munchen, Planegg-Martinsried, Germany; (3) Heinrich-Heine-Universität, Düsseldorf, Germany

Extracellular vesicles (EVs) are ubiquitous mediators of intercellular communication. Growing evidence shows that EVs produced by plant pathogens carry modulators of plant immunity, also in the form of RNA effectors. The maize smut fungus, Ustilago maydis, is an archetypal smut pathogen and an established model for fungal RNA transport. We aim to isolate and characterise the RNA cargo carried by EVs of $U$. maydis to identify potential RNA effectors relevant for infection. EV isolation methods were developed for axenic cultures of $U$. maydis and infected maize leaves. U. maydis EV-associated RNA, protected from RNase treatment, were found to include various full-length mRNA and tRNA. RNA-seq analysis was carried out on EVs from axenic cultures of induced filaments, which partially mimic the transcriptional profile of the early infection stage. This revealed the presence of full-length mRNAs, including those from previously defined infection-related expression modules and effector gene clusters, prompting further investigation of fungal EVs in planta. Furthermore, an EV marker is being developed for improved purification and visualisation of $U$. maydis EVs from infected maize. Examination of fungal EVs from infected maize tissues is currently underway to identify and determine the fate of promising mRNA cargos during infection.

Distinct flavonoids in Cicer arietinum regulate positive and negative interactions with Mesorhizobium ciceri and Meloidogyne javanica J. L. P. NG, H. Zainal Abidin, U. Mathesius, The Australian National University, Acton, ACT, Australia

Legumes can form symbioses with nitrogen-fixing rhizobia. Nodulation begins when host roots exude flavonoids to attract and induce compatible rhizobia. Flavonoids are also necessary for nodule development. In the field, however, rhizobia do not interact with legumes alone. Plant-parasitic 
nematodes (PPNs), e.g. root-knot nematodes (RKNs) are very successful gall-forming parasites that infect a wide host range, including legumes. Chickpea (Cicer arietinum) is the second most widely grown legume crop in the world. Mesorhizobium ciceri is the natural rhizobial symbiont of chickpea and Meloidogyne javanica is a broad host-range RKN that successfully infects chickpea. We hypothesise that distinct flavonoids are involved in the chemoattraction of rhizobia and RKNs and development of nodules or galls, respectively. Previous studies suggested some flavonoids could act as nematostatic and nematicidal compounds against RKNs, affecting their motility, gall and egg formation. I will present data showing how some flavonoid compounds could serve as attractants/repellents for both $M$. ciceri and $M$. javanica, as well as regulating nodulation and gall development, respectively. Current data show that chrysin, 2-hydroxyflavone and 3-hydroxyflavone are potential flavonoid markers for high nodulation, whereas increased daidzein concentrations could reduce gall formation. Our goal is to establish flavonoid markers in chickpea that are indicative of high nodulation and tolerance towards PPNs.

\section{A TIR-NBS-LRR protein is necessary for BAK1 autoimmune phenotypes and links BAK1-mediated cell death to effector triggered immunity}

L. YU (1), S. Schulze (1), J. Imkampe (1), T. Halter (1,2), V. Cevik (3), B. Kemmerling (4), (1) ZMBP, University of Tuebingen, Tuebingen, Germany; (2) Institut de Biologie de l'Ecole Normale Supérieure (IBENS), Paris, France; (3) Department of Biology \& Biochemistry, University of Bath, Bath, U.K.; (4) ZMBP, University of Tuebingen, Tübingen, Germany

The membrane-localized co-receptor BRASSINOSTEROID INSENSITIVE1-ASSOCIATED RECEPTOR KINASE1 (BAK1/SERK3), regulates different signaling pathways including growth and development, immune response, and cell death control by directly interacting with and positively regulating multiple ligand binding receptors. The BAK1-interacting RK BIR3 can prevent BAK1-ligand binding receptor interaction by directly interacting with both ligand-binding receptors and BAK1 (and all the members of SERK family). The interaction of BIR3 with SERKs stabilizes BAK1 and its closest homolog BKK1/SERK4. The interactome of BIR3 revealed a BIR3 interacting TIR-NBS-LRR (TNL) protein (BIT1). Double mutants in bitl bakl show reduced cell death compared to bakl single mutants upon inoculation with the necrotrophic fungus Alternaria brassicicola. Double mutants in bir3 bakl show a severe dwarf phenotype and spontaneous cell death. Our investigations revealed that mutations in bitl also suppress bir 3 bakl-mediated cell death. Both bakl and bir3 bakl-mediated cell death can be partially suppressed by mutations in ENHANCED DISEASE SUSCEPTIBILITY (eds1), a downstream component of TNLs. Taken together, BIT1 interacts with BAK1 and BIR proteins, is necessary for BAK1-mediated cell death and links BAK1 to TIR-NBS-LRR mediated cell death usually involved in effector triggered immunity. BIT1 likely guards the integrity of BAK1 and BAK1 BIR complexes and initiates autoimmune cell death when BAK1 complexes are impaired.

"Disease-climate-microbiome" interactions in the phyllosphere

S. Y. HE, Michigan State University, U.S.A.

Howard Hughes Medical Institute, East Lansing, MI, U.S.A.

One of the most significant challenges of the 21 st century is to discover innovative ways of increasing global crop production to meet the demands for food from the growing human population in the context of changing climate. Greater efforts are needed to accelerate the buildup of a comprehensive knowledge base that explains how plant diseases occur; how plants defend against pathogens; and how dynamic climate conditions impact plants, microbes, and their interactions. The famous "Disease Triangle" concept states that plant disease outbreaks require not only a susceptible plant and a virulent pathogen, but also conducive environmental conditions. For practical reasons, however, most contemporary investigations into plant-pathogen interactions at the molecular level devote relatively little effort to understanding why climatic conditions, such as humidity and temperature, have a profound effect on pathogen virulence and host susceptibility. Moreover, these studies often ignore the potentially pervasive effect a plant's endogenous microbiome may have on basic plant health and host-pathogen interactions. In this talk, I will give an example of interplays between disease, environment and microbiota in Arabidopsis thaliana leaves. Our work suggests that future studies of plant-pathogen interactions should consider the multi-dimensional nature of "disease-environment-microbiome" interactions in order to capture novel phenomena and missing links in plant-microbe interactions.

Emerging roles of the root microbiota in balancing plant growth and defense for microbe-host homeostasis K. W. MA (1), Y. Jia (1), J. Ordon (1), H. Nakagami (2), R. Garrido-Oter (1), P. Schulze-Lefert (1), (1) Max Planck Institute for Plant Breeding Research, Cologne, Germany; (2) Max Planck Institute for Plant Breeding Research, Germany

The plant immune system, including MAMP-triggered immunity (MTI), restricts microbial proliferation, but activation of plant defense responses generally comes at the price of plant growth. Emerging evidence suggests that both commensal and pathogenic microbes can modulate plant immune responses. Here, we surveyed the capacity of members of a bacterial Arabidopsis root culture collection ( $A t$-R-sphere) to suppress MAMP-elicited root growth inhibition (RGI). Surprisingly, $43 \%$ of At-R-sphere members, representing bacterial species from four phyla, suppressed RGI under exogenous MAMP/DAMP stimulation. In contrast, specific A $t$-R-sphere microbes activated immunity and induced RGI without flg22 stimulation. By comparing taxonomically equivalent RGI-suppressive or non-suppressive synthetic communities (SynComs), we showed that RGI-suppressive activity is a dominant community trait and associated with downregulation of immune responses. Biochemical and genetic analyses indicate that lineage-specific mechanisms different from known virulence mechanisms are deployed by microbiota members to interfere with MAMP-triggered responses. Our results suggest that the plant innate immune system exerts selection pressure on both bacterial commensals and pathogens to maintain distinct immunosuppression traits. Further dissection of the host pathway(s) involved might identify components that could be targeted to uncouple or bypass the trade-off between plant growth and defense.

TIR Domains of Plant Immune Receptors are NAD+ Consuming Enzymes that Promote Cell Death M. T. NISHIMURA, Colorado State University, Department of Biology, Fort Collins, CO, U.S.A.

Plant nucleotide-binding leucine-rich repeat (NLR) immune receptors activate cell death and confer resistance to pathogens. The mechanism by which plant NLRs trigger cell death remains obscure. We demonstrate that plant TIR (Toll/interleU.K.in-1 receptor) domains are enzymes 
capable of degrading NAD'. Both cell death induction and NADase activity of plant 'TIR-only' proteins and TIR domains derived from TIRNBS-LRR (TNL) immune receptors require known self-association interfaces and a putative catalytic glutamic acid conserved in both bacterial TIR NADases and the mammalian SARM1 TIR NADase. We identify a novel TIR-dependent NAD ${ }^{+}$breakdown product in vitro and use it as an in planta biomarker of TIR enzymatic activity. We show that TIR enzymatic activity is induced by pathogen effector recognition, and functions upstream of EDS1 and NRG1, two proteins required for TIR immune function. Thus, plant TIR-NLR receptors require a conserved enzymatic function to transduce recognition of pathogens into a cell death response.

\begin{abstract}
A hierarchical transcriptional network controls appressorium development in the rice blast fungus Magnaporthe oryzae in response to surface hydrophobicity

M. OSES-RUIZ (1), M. Martin-Urdiroz (2), D. Soanes (2), M. J. Kershaw (2), G. Littlejohn (3), N. Cruz-Mireles (1), P. Derbyshire (1), F. L. H. Menke (1), N. J. Talbot (1), (1) The Sainsbury Laboratory, University of East Anglia, Norwich Research Park, Norwich, U.K.; (2) University of Exeter, U.K.; (3) University of Plymouth, Plymouth, U.K.

Rice blast disease is one of the most destructive diseases of cultivated rice. It is caused by the ascomycete fungus Magnaporthe oryzae. Depending on the nature of the contact surface, $M$. oryzae undergoes distinct morphogenetic programs mediated by the Pmk1 MAPK signaling cascade that acts on a set of downstream transcription factors that rewire specific morphogenesis-associated gene expression programs. Here, we show how an inductive hydrophobic surface leads to formation of the appressorium, the infection structure required for plant infection We show fundamental differences between both developmental programs and how these are linked to cell cycle regulation and type II autophagic cell death. We also show that surface recognition is tightly linked to activation of the Pmk1 MAPK and its phosphorylation of targets leading to a downstream transcriptional response. We report how comparative transcriptomic analysis has been used to investigate the main genetic circuitry associated with appressorium morphogenesis. We show that appressorium development and invasive growth are controlled by a co-regulated set of 15 transcription factors, that are dependent on the Pmk1 MAPK pathway. Moreover, we show how a specific homeobox transcription factor called Hox7, which is a direct target of Pmk1, acts as a regulator of autophagy, cell cycle arrest and suppression of hyphal-associated gene expression, that are necessary for appressorium development and septin-dependent plant infection.
\end{abstract}

Co-evolutionary diversification of barley MLA immune receptors by direct recognition of sequence-unrelated powdery mildew AVRA effectors

M. L. SAUR (1), T. Maekawa (1), S. Bauer (1), B. Kracher (2), L. Frantzeskakis (3), R. Panstruga (3), P. Schulze-Lefert (1), (1) Max Planck Institute for Plant Breeding Research, Cologne, Germany; (2) Max Planck Institute for Plant Breeding Research, Germany; (3) RWTH Aachen University, Aachen, Germany

Disease resistance is mediated by recognition of pathogen avriulence effectors (AVR) through host nucleotide-binding leucine-rich repeat receptors (NLR). The barley (Hordeum vulgare) mildew locus $A$ (mla) encoded NLRs (MLAs) confer isolate-specific resistance to the widespread mildew fungus Blumeria graminis forma specialis hordei $(\mathrm{Bgh})$. Mla orthologs $\mathrm{Sr} 33$ and $\mathrm{Sr} 50$ confer resistance to the stem rust fungus Puccinia graminis f. sp. tritici (Pgt) in wheat. In barley, Mla has been subject to extensive functional diversification at their LRR domains, resulting in allelic resistance specificities, each recognizing a cognate $B g h A V R_{a}$. We isolated $B g h A V R_{a l}, A V R_{a 7}, A V R_{a 9}, A V R_{a 13}, A V R_{a 10}$, and $A V R_{a 22}$, which encode small secreted proteins recognized by allelic barley MLA1, MLA7, MLA9, MLA13, MLA10, and MLA22 receptors, respectively. We connect $A V R_{a}$ genomic location with previous linkage studies and show the ability of $\mathrm{AVR}_{\mathrm{A}}$ effectors to induce cognate MLAspecific cell death in barley, Arabidopsis thaliana and Nicotiana benthamiana. The isolated $A V R_{a}$ effectors are sequence-unrelated, except for allelic $A V R_{a 10}$ and $A V R_{a 22}$ that are co-maintained in pathogen populations in the form of a balanced polymorphism. Despite the lack of detectable similarity amongst effectors recognised by MLA, co-expression experiments with matching Mla-AVR $R_{a}$ pairs indicate direct detection of the sequence-unrelated fungal effectors by MLA receptors.

Apoplastic recognition of microbial Nep1-like proteins (NLPs) and subsequent signaling leading to plant immunity T. Raaymakers, S. Lambregts, G. VAN DEN ACKERVEKEN, Plant-Microbe Interactions, Dept of Biology, Utrecht University, Utrecht, Netherlands

Necrosis- and ethylene-inducing peptide 1 (Nep1)-like proteins (NLP) occur in bacteria, fungi, and oomycetes, in particular in plant-associated species. Plant pathogenic microbes secrete NLPs into the apoplast where they can either act as cytotoxins (only in eudicot but not monocot plants) or be non-cytotoxic. Several plant species have evolved the capacity to recognize NLPs as molecular patterns and trigger plant immunity. In Arabidopsis, a receptor complex consisting of RLP23-SOBIR1-BAK1 mediates NLP-triggered immunity (NTI) in Arabidopsis. Here, we present our data on the genetic dissection of NTI, using a transgenic Arabidopsis line expressing an estradiol-inducible version of HaNLP3 of the downy mildew Hyaloperonospora arabidopsidis. We have obtained a number of decreased $\underline{N T I}$ (dni) mutants that also no longer respond to the 24 amino acid NLP peptide nlp24. We will report on our progress on $d n i$ mutant analysis and cloning of the corresponding DNI genes.

The evolution of agrobacteria and their oncogenic plasmids

J. Weisberg (1), E. Davis (1), J. Tabima (1), B. J. Knaus (1), W. Ream (1), M. S. Belcher (1), M. Miller (1), C. H. Kuo (2), E. M. Lai (2), J. E. Loper (1), N. J. Grunwald (3), M. L. Putnam (1), J. H. CHANG (1), (1) Oregon State University, Corvallis, OR, U.S.A.; (2) Academia Sinica, Taipei, Taiwan; (3) USDA ARS, Corvallis, OR, U.S.A.

Agrobacteria are plant- and soil-associated bacteria with an unusual multipartite genome architecture. Strains carrying large oncogenic plasmids can genetically transform plants to establish a nutrient-replete niche and cause crown gall and hairy root diseases. A small number of agrobacterial strains and oncogenic plasmids have been disarmed and engineered into powerful biological tools that revolutionized approaches for plant biology. To better manage their diseases and expand their uses for biotechnology, it is critical to understand the evolutionary processes that shaped agrobacteria and their oncogenic plasmids. To this end, we determined the genome sequences for 162 strains collected from 19272016, six different continents, and 52 species of plants, primarily showing crown gall disease. The replicons were characterized in a dataset of 
over 1,000 genome sequences to model the emergence of agrobacteria from within a larger group that includes multiple groups of rhizobia. We also classified oncogenic plasmids and determined the principles governing their organization to reconstruct the evolution of the genetic determinants necessary for executing the extraordinary process of inter-kingdom gene transfer. Findings provide a framework to guide the taxonomic classification of agrobacteria, epidemiological studies of these pathogens, and the optimization of agrobacteria and their plasmids for use in plant biology and biotechnology.

Temporal transcriptome profiling reveals distinct gene expression signatures associated with suppression of plant immunity by high temperature and high humidity

A. MINE (1,2), A. Matsumoto (3), A. Takeda (1), (1) Department of Biotechnology, College of Life Sciences, Ritsumeikan University, Kusatsu, Shiga, Japan; (2) PRESTO, Japan Science and Technology Agency, Kawaguchi-shi, Saitama, Japan; (3) Research Organization of Science and Technology, Ritsumeikan University, Kusatsu, Shiga, Japan

High temperature and high humidity often cause outbreaks of plant diseases in agricultural fields. However, the molecular mechanisms behind these biological phenomena are poorly understood. Arabidopsis thaliana Col-0 is susceptible to Pseudomonas syringae pv. tomato DC3000 (Pto) but resistant to Pto carrying AvrRpt2 or AvrRpm1 effector due to activation of effector-triggered immunity. Here, we investigated transcriptome dynamics of A. thaliana Col-0 with twelve time points after challenge with Pto and Pto carrying AvrRpt2 or AvrRpm1 under standard laboratory condition, high temperature and high humidity. Resistance against Pto and Pto carrying AvrRpt2 or AvrRpm1 were negatively affected by high temperature and high humidity to varying extents. Co-expression network analysis revealed modules of co-expressed genes whose expression levels were comparable at early time points but compromised later on under high temperature in comparison to standard laboratory condition. We employed dynamic Bayesian networks to model regulatory relationships between co-expression modules under the three different environmental conditions. Comparative analysis of predicted regulatory relationships pointed to high humidity-specific misregulation of pattern recognition receptors, receptor-like proteins and immune-related transcription factors. Thus, our results indicate that distinct gene expression signatures are associated with immune suppression by high temperature and high humidity.

Systematic discovery of antimicrobial proteins employed by the plant microbiome M. GELLER, I. Pollin, A. Levy, The Hebrew University of Jerusalem, Rehovot, Israel

The plant environment is teeming with microbes that are attracted to the rich nutrients exuded by the plant host. In order to colonize the highly occupied niche of the rhizosphere, bacteria have ostensibly evolved various mechanisms to hinder microbial competitors. These mechanisms play a role in microbial community assembly at the rhizosphere and the endosphere. However, there is a little understanding of the identity of these molecular mechanisms, their targets, and the actual molecules used to inhibit microbial growth in the plant environment. We study two bacterial secretion systems that are commonly employed by members of the plant microbiome named the Type 6 Secretion System (T6SS) and the Phagelike Translocation Structure (PLTS), both of which are derived from bacteriophage tails. We developed a computational algorithm and used it to scann thousands of bacterial genomes and to predict novel proteins (effectors) secreted by each of the secretion systems. We then validated the antimicrobial activity of selected putative effectors through heterologous expression in various microbial cells. Our results have uncovered seven novel antimicrobial proteins families, shedding light on new mechanisms by which bacteria gain a selective advantage in the plant environment. Harnessing these mechanisms in different agricultural settings can lead to efficient, sustainable biocontrol strategies.

Phosphate status-dependent control of associations with beneficial and pathogenic fungi in Arabidopsis thaliana T. H. Lee (1), T. Inoue (1), M. Tanaka (1), T. Hirase (1), S. Yasuda (1), K. Hiruma (1,2), Y. SAIJO (1), (1) Nara Institute of Science and Technology, Japan; (2) JST PRESTO, Japan

Under phosphate (Pi) deficiency, plants often rely on root-associated fungi for Pi nutrition, while retaining plant immunity against pathogens. However, how plant immunity, microbial infection strategies and plant-microbe associations are modulated according to Pi availability remains poorly understood. By exploiting the root-colonizing plant growth promoting fungus Colletotrichum tofieldiae $(\mathrm{Ct})$ and its pathogenic relative, $C$. incanum (Ci), we pursue this question in Arabidopsis thaliana. Under low Pi, $\mathrm{Ct}$ and $\mathrm{Ci}$ both deplete the Leucine-rich-repeat receptor kinase BAK1 in roots to promote their infection. RNA-sequencing analyses in root responses to fungal chitin and the endogenous Pep1 peptide reveal that Pep1 induction, but not chitin induction, of defense-related genes is elevated under low Pi. This sensitization requires Pep receptors (PEPRs), the receptor-like cytoplasmic kinase BIK1 and the ferroxidase LPR1/LPR2-mediated Pi starvation response (PSR). Importantly, Ci pathogen resistance is impaired in plants lacking PEPR and PSR pathways, indicating that PSR-mediated reinforcement of PEPR defenses serves to maintain pathogen resistance under low Pi. Conversely, Ct-mediated plant growth benefits require suppression of PEPR defenses. Our findings suggest that the interplay between PSR signaling and PEPR-mediated damage signaling is critical in establishing beneficial association with mutualistic fungi while retaining pathogen resistance.

A cyst nematode effector counteracts host immunity by directly binding redundant nodes in an NLR immune receptor network L. DEREVNINA (1), C. H. Wu (1), H. Adachi (1), A. Maqbool (1), R. Xie (1), J. Upson (1), A. Goverse (2), S. Kamoun (1), (1) The Sainsbury Laboratory, University of East Anglia, Norwich Research Park, Norwich, U.K.; (2) Laboratory of Nematology, Wageningen University and Research, Wageningen, Netherlands

Plant pathogens deploy effectors that target multiple layers of immune networks. However, the mechanisms by which effectors counteract immunity, particularly NLR receptor networks, remain poorly understood. We investigated the degree to which pathogens suppress the NRC network in which a large number of sensor NLRs converge to function through the partially redundant helper NLRs NRC2, NRC3 and NRC4. We screened effectors from oomycetes, bacteria, aphids and nematodes for suppression of NRC-mediated immunity and identified SS15-a SPRYSEC-type effector from the potato cyst nematode Globodera rostochiensis - as a suppressor of NRC2 and NRC3 but not NRC4. SS15 suppresses autoactive NRC2/3 indicating that it functions independently of sensor NLRs. Multiple protein-protein interaction assays, including yeast two-hybrid, in planta co-immunoprecipitations and in vitro gel filtration, revealed that SS15 directly interacts with the nucleotide binding 
(NB) domain of NRC2 and NRC3. Binding to the suppressed NRCs occurred with stronger affinity relative to NRC4.We are currently using biochemical and cellular approaches to understand how SS15 binding perturbs NRC activities. Our work sheds light on the arms race dynamics between pathogens and NLR networks. We can use this knowledge to generate receptor variants that evade pathogen suppression. This should prove particularly useful when the pathogen-targeted receptor is a critical node in the immune network as in the case of the NRCs.

\section{A fundamental role of microbiota in age-dependent immunity}

B. C. PAASCH (1), J. M. Kremer (1), K. Nomura (1), B. H. Kvitko (2), C. A. Thireault (1), F. T. Dion (1), T. A. Ulrich (1), C. E. Griffin (1), D. D. Rhodes (1), J. M. Tiedje (1), S. Y. He (3,4), (1) Michigan State University, East Lansing, MI, U.S.A.; (2) University of Georgia, Athens, GA, U.S.A.; (3) Michigan State University, U.S.A.; (4) Howard Hughes Medical Institute, East Lansing, MI, U.S.A.

A diverse community of microorganisms inhabits the tissues of healthy plants. To help restrict the growth of opportunistic and pathogenic microorganisms, plants have evolved a two-tiered innate immune system capable of eliciting immune responses upon the recognition of broadly conserved pathogen-associated molecular patterns (PAMPs) or specific pathogen-secreted virulence effectors. Like newborn animals, many plants exhibit age-dependent immunity whereby innate immune responses become more robust over time after germination. A large number of studies have shown that specific microbes can ectopically enhance immune responses of plants that are already colonized by endogenous microbiota. However, the role of preexisting endogenous microbiota in the development of immunity in plants remains a fundamental question, especially when plants are grown in soil substrates. Using a newly developed, soil-based gnotobiotic plant growth system, we found that Arabidopsis plants grown in the absence of microbiota are essentially immune-deficient and hyper-susceptible to bacterial infection compared to plants colonized by natural microbial communities. Strikingly, germ-free plants lost age-dependent immunity, which could be reconstituted by introduction of natural soil microbiota. Our study uncovers a fundamental role of microbiota in age-dependent immunity, which was previously thought to be an intrinsic trait of plants.

Cleavage of bacterial MucD by plant secreted proteases in Arabidopsis immunity Y. WANG (1), R. Garrido-Oter (1), J. Wu (2), T. M. Winkelmüller (1), M. Agler (3), T. Colby (1), E. Kemen (1), K. Tsuda (1), (1) Max Planck Institute for Plant Breeding Research, Cologne, Germany; (2) Institute of Plant Physiology \& Ecology, Chinese Academy of Sciences, Shanghai, China; (3) Max Planck Institute for Plant Breeding Research, Germany

During the past three decades, researchers have focused on the recognition of pathogen molecules, activation of plant signaling pathways and defense genes required for plant immunity. These signal pathways explain how plants relay information for pathogen recognition to downstream signaling events within the plant cells. However, the fundamental question "how do plant immune responses suppress growth of bacterial pathogens in plants?" was not answered. Here, we provide compelling biochemical and genetic evidence for a mechanism by which plant immunity directly suppresses in planta growth of bacterial pathogens. Two Arabidopsis thaliana Secreted Protease, SAP1 and SAP2, cleave the evolutionarily conserved bacterial protein MucD to redundantly inhibit growth of the bacterial pathogen Pseudomonas syringae. Antibacterial activity of SAP1 required its protease activity in planta and in vitro. SAP1 overexpression led to enhanced MucD cleavage and plant resistance without penalties in plant growth and reproduction, while mutant plants deficient in both SAP1 and SAP2 exhibited compromised MucD cleavage and resistance. $P$. syringae $\triangle m u c D$ showed compromised growth in planta and in vitro. Notably, growth of $\triangle m u c D$ complemented with $\mathrm{MucD}^{\mathrm{F} 106 \mathrm{Y}}$, which SAP1 was unable to cleave, was not affected by SAP activity. Our findings provide compelling evidence for an antibacterial mechanism in plants.

Insights into the sites of action of ALD1 using chimeric Arabidopsis with epidermal ALD1

J. T. GREENBERG (1), S. C. Jiang (1), N. M. Cecchini (2,3), Z. Banday (1), N. Engle (4), T. Tschaplinski (4), (1) University of Chicago, Chicago, IL, U.S.A.; (2) CIQUIBIC-CONICET, Dpto de Química Biológica-Ranwel Caputto, Cordoba, Argentina; (3) CIQUIBIC-CONICET, Universidad Nacional de Córdoba, Cordoba, IL, Argentina; (4) Oak Ridge National Laboratory, Oak Ridge, TN, U.S.A.

The Arabidopsis plastid-localized aminotransferase ALD1 is needed for bacterial disease resistance associated with infections of both naïve plants as well as those that have previously been infected, a process that leads to systemic acquired resistance (SAR). ALD1 is in the pathway that produces pipecolic acid (Pip) and N-hydroxy-Pip in response to pathogens. Additionally, ALD1 is associated with the production of a non-Pip basal metabolite present before infection. We constructed plants in which ALD1 was expressed only in epidermal cells of leaves treated with an inducer. Local disease resistance was fully restored when ALD1 was induced in epidermal cells. The response-gain of SAR was restored in plants with ALD1 produced only at the immunization site. Although Pip in the lower infected leaves was restored, Pip did not accumulate in the distal tissue during SAR activation of the chimeric plants. ALD1 and Pip at the immunization site may permit the local infected tissue to reach a high enough signaling threshold to induce SAR. However, the absolute disease resistance of distal leaves was lower than in WT. We propose that ALD1 is required at the immunization site to induce SAR activation, but basal ALD1 (and possibly a non-PIP metabolite(s)) is also needed at the secondary, distal infection site to fully suppress pathogen growth. Our findings suggest a critical role for epidermal plastids and the plastidlocated protein ALD1 in local and whole plant immune signaling.

Ethylene signaling mediates fine-tuning of host infection by parasitic plants S. Cui (1,2), T. Kubota (1), Y. Yoshimura (1), K. Shirasu (2,3), S. YOSHIDA (1,2), (1) NAIST, Nara, Japan; (2) RIKEN Center for Sustainable Resource Science, Yokohama, Japan; (3) The University of Tokyo, Tokyo, Japan

Parasitic plants in Orobanchaceae, such as Striga and Orobanche spp., are among the most devastating agricultural pests. However, the molecular mechanisms underlying their host invasion have not been well understood. Parasitic plants in Orobanchaceae form an invasive organ, the haustorium, on their roots. The haustorium penetrates into host tissues, reaches the host steel, and connects their vasculatures with those of hosts to acquire nutrients and water. To elucidate how parasitic plants invade host tissues, we have employed forward genetic approaches using a model parasitic plant, Phtheirospermum japonicum. Mutant screening of $P$. japonicum identified two mutant lines that are defective in host infection. In wild type $P$. japonicum, haustorium apex cells differentiate into tissue-invading intrusive cells when they reach to host root surface, 
while these mutants often fail to develop intrusive cells and grow around host roots without penetration. In addition, these mutants show elongated shapes of haustoria when exposed to haustorium-inducing signals, such as 2,6-dimethoxy-p-benzoquinone, even in the absence of host plants. We have identified the causal genes for these mutants using genome analysis and re-sequencing. These mutants have defects in the key components of ethylene signaling. Our results indicate that ethylene signaling regulates cell fates of haustorium apex cells to fine-tune the haustorium length and enable precise invasion into host tissues.

Revealing the unknown: The isoelectric point of proteins influences their translocation into the extrahaustorial matrix L. SMIGIELSKI (1), G. B. Aguilar (1), M. Kwaaitaal (1,2), H. Thordal-Christensen (1), (1) University of Copenhagen, Frederiksberg C, Denmark; (2) University of Amsterdam, Amsterdam, Netherlands

The obligate biotrophic powdery mildew fungus, Blumeria graminis, is a model pathogen suitable for studies of haustorial function, including those of the extrahaustorial membrane (EHM) and matrix (EHMx). Here the communication between the plant and the fungus takes place: effectors are delivered, nutrients taken up and other processes are coordinated. Despite the importance of this intersection, not much is known about it. Recent studies show that the EHM has ER-like properties, and that ARA6, a protein localizing at multivesicular bodies, can be found at this membrane as well.

We used particle bombardment to investigate the localization of altered fluorescent proteins expressed in single barley cells infected with powdery mildew. The extent by which proteins were able to cross the EHM was studied by confocal microscopy. Amino acids were attached to mCherry and mYFP to create proteins of the same size, but with different overall $\mathrm{pI}$ values, and we could show that translocation is favored by $\mathrm{pl}$ between 6.0 and 8.4. However, protein size and sequence properties also appear to play a role. We suggest that an EHM translocon might be responsible for protein uptake into the EHMx.

Our insight into this important point of contact between the plant and the fungus might guide further investigations that can assist us in exploiting these properties with the ultimate goal of protecting crops from the powdery mildew fungus, and thus, to reduce yield losses.

Calcium signaling in a compatible biotrophic oomycete-plant interaction

F. Y. HWU, M. Parniske, Genetics, Faculty of Biology, University of Munich (LMU), Biocenter Martinsried, Martinsried, Germany

A hallmark of arbuscular mycorrhiza and the nitrogen-fixing root nodule symbiosis is the accommodation of fungal and bacterial partners inside living plant cells. The biotrophic compatible interaction between the oomycetal pathogen Hyaloperonospora arabidopsidis (Hpa) and Arabidopsis involves the formation of haustoria, structures cytologically related to arbuscules. We are interested to what extend signal transduction processes mediating intracellular plant root symbioses also play a role in the Arabidopsis/Hpa interaction. Although Arabidopsis lost the ability to form arbuscular mycorrhiza during evolution, the genome still carries genes closely related to legume genes essential for symbiotic signal transduction, like the nuclear-localised cation channel POLLUX or homologs of the symbiosis receptor-like kinase SYMRK (ShRKs). We observed calcium waves and oscillations in leaves heavily infected with Hpa. In leaves of mutants of POLLUX and ShRKs these oscillations were altered and Hра produced fewer sporangiophores and an increased frequency of multilobed haustoria. Transcriptome analysis of $H p a$ infected wild-type and mutant leaves revealed gene expression signatures related to calcium signaling to be altered in pollux and shrk mutants. We present a mechanistic model for the role calcium signaling in the compatible interaction of Arabidopsis with its oomycetal pathogen.

Keywords: Calcium signaling, compatible interaction, Arabidopsis thaliana, Hyaloperonospora arabidopsidis.

The role and regulation of plastid targeting of AZI1, a key factor for systemic immunity D. J. SPEED (1), N. M. Cecchini (2,3), S. Roychoudhry (4), J. T. Greenberg (1), (1) University of Chicago, Chicago, IL, U.S.A.; (2) CIQUIBICCONICET, Dpto de Química Biológica-Ranwel Caputto, Cordoba, Argentina; (3) CIQUIBIC-CONICET, Universidad Nacional de Córdoba, Cordoba, IL, Argentina; (4) University of Leeds, Leeds, U.K.

The proper subcellular localization of defense factors is critical to plant immune responses. A key component for systemic resistance, lipid transfer protein (LTP)-like AZI1, is needed for the systemic movement of the priming signal azelaic acid (AZA) that derives from plastid envelopes. A pool of AZI1 traffics to the plastid envelope membrane as well as other membranes such as the plasma membrane and the ER. After systemic defense-triggering infections or in a constitutively defensive mutant, the proportion of AZI1 localized to plastids increases in a manner that depends on defense-related kinases MPK3 and MPK6. MPK3 and MPK6 are also required for AZA-induced priming, which suggests the plastid pool of AZI1 is involved in defense. Intriguingly, AZI1 does not possess a classical plastid transit peptide or typical features of signalanchored proteins that can explain its plastid localization. Using confocal microscopy and fractionation, we studied the sequence requirements for proper localization of AZI1 to plastids. We show that AZI1 belongs to a unique class of signal-anchored proteins that uses a bipartite N-terminal signature that allows for its plastid targeting. Our results indicate the existence of a novel mode of plastid targeting that is implicated in defense responses. Furthermore, since MPK3/6 regulate AZI1's targeting/trafficking, these kinases in turn might determine how much defense signal(s) moves systemically for resistance and priming induction.

Nutrient status regulates plant recognition of signals from beneficial microbes for promotion of symbiosis and suppression of immunity F. FENG (1), J. Sun (1), T. Lee (1), G. V. Radhakrishnan (2), Z. Bozsoki (3), S. Radutoiu (3), J. Stougaard (3), G. Oldroyd (1), (1) Sainsbury Laboratory Cambridge University, Cambridge, U.K.; (2) John Innes Centre, Norwich, U.K.; (3) University of Aarhus, Aarhus, Denmark

Plant roots associate with myriads of microorganisms in the rhizosphere, which can have detrimental or beneficial effects on plant survival. The current understanding is that plants control microbial invasion through activation of immunity signaling by microbial-associated molecular patterns (MAMPs) and activation of symbiosis signaling by short chain chitooligosaccharides and lipochitooligosaccharides, to either restrict or promote microbial associations, respectively. It is thought that the differential activation of these pathways, by differential elicitors, explains the plants' ability to differentiate between pathogens and symbionts. However, we demonstrate that MAMPs present on microbial surfaces that activated immunity signaling, also activate symbiosis signaling in Medicago truncatula to facilitate colonisation by beneficial mycorrhizal fungi. Symbiosis signaling in Medicago is enhanced under nutrient starvation, while immunity signaling is suppressed. Our work suggests that plants do 
not differentiate a pathogen from a symbiont simply through differential elicitor recognition alone, rather combinations of differential elicitors, along with the plants' nutrient status, combine to define a symbiotic or immunogenic outcome.

Glycosidase and glycan polymorphism control hydrolytic release of immunogenic flagellin peptides

P. Buscaill (1), B. Chandrasekar (1), N. Sanguankiattichai (1), J. Kourelis (1), F. Kaschani (2), E. L. Thomas (1), K. Morimoto (3), M. Kaiser (2), G. M. Preston (1), Y. Ichinose (4), R. A. L. VAN DER HOORN (3), (1) University of Oxford, U.K.; (2) University of Duisburg-Essen, Germany; (3) Department of Plant Sciences, University of Oxford, Oxford, U.K.; (4) Okayama University, Okayama, Japan

Plants and animals recognise conserved flagellin fragments as a signature of bacterial invasion. These immunogenic elicitor peptides are embedded in the flagellin polymer and require hydrolytic release before they can activate cell surface receptors. Although much of flagellin signaling is understood, we know little on the release of immunogenic fragments. We discovered that the plant-secreted $\beta$-galactosidase-1 (BGAL1) of Nicotiana benthamiana promotes hydrolytic elicitor release and acts in immunity against pathogenic Pseudomonas syringae strains. BGAL1 only acts on strains that carry a terminal modified viosamine (mVio) in the flagellin $O$-glycan. In counter defence, various $P$. syringae pathovars evade host immunity by using BGAL1-resistant $O$-glycans or by producing a BGAL1 inhibitor. I will discuss the discovery of BGAL1, the implications from this work including the role of BGAL1 orthologs and our approaches to identify the BGAL1 inhibitor.

S-acylation-Greasing the mechanisms of plant immunity

C. H. Hurst (1), D. Turnbull (1), P. A. HEMSLEY (1,2), (1) University of Dundee, Dundee, U.K.; (2) The James Hutton Institute, Dundee, U.K.

S-acylation is a fatty acid based post-translational modification of proteins thought to alter a protein's physical properties leading to alterations in protein function, localisation, turnover or interactions. It is unique amongst lipid modifications of proteins as it is reversible; this lends it the ability to act as a regulatory mechanism. We have shown that $\sim 40 \%$ or the plant membrane proteome is S-acylated and that S-acylation affects both integral and peripheral membrane proteins. The study of S-acylation is still in its infancy, but we have found that S-acylation is key for the function of a number of plant defence processes including the receptor-like kinase superfamily. Receptor-like kinases are responsible for perception of almost all extracellular physical stimuli in plants and are the front line of defense against pathogens. The S-acylation state of RLKs rapidly changes upon ligand biding and we will discuss our novel findings in the context of RLK signaling regulation. We will also detail our work on defining the dynamics and regulation of the S-acyl proteome to increase understanding of how this enigmatic modification regulates membrane protein function during plant-microbe interactions.

Convergent evolution of Pik-1 immune receptors towards high-affinity binding of an effector from the rice blast fungus

A. BIALAS (1), T. Langner (1), M. P. Contreras (1), R. Kellner (1,2), R. Terauchi (3,4), M. J. Moscou (1), M. J. Banfield (5), S. Kamoun (1), (1) The Sainsbury Laboratory, University of East Anglia, Norwich Research Park, Norwich, U.K.; (2) Max-Planck Institute for Plant Breeding Research, Cologne, Germany; (3) Graduate School of Agriculture, Kyoto University, Kyoto, Japan; (4) Iwate Biotechnology Research Center, Kitakami, Japan; (5) John Innes Centre, Norwich Research Park, Norwich, U.K.

The Oryza genus has evolved the Pik-1 and Pik-2 proteins - a pair of NLR immune receptors that confer disease resistance to the rice blast fungus, Magnaporthe oryzae. Although the Pik-2 protein has a typical NLR domain architecture, Pik-1 carries an unconventional Heavy MetalAssociated (HMA) domain that directly binds the M. oryzae AVR-Pik effector. We aim to understand the evolutionary history of the Pik-1 receptor and test hypotheses about the adaptive evolution of its integrated HMA domain. Phylogenetic analyses of Pik orthologues indicated that the HMA domain integrated into Pik-1 nearly 50 million years ago. Ancestral HMA sequence reconstruction coupled with functional studies revealed that different allelic variants of Pik-1—Pikp-1 and Pikm-1 — convergently evolved to recognise AVR-PikD. Using biophysical approaches, we functionally characterised two regions in the Pik-1 HMA that independently evolved towards high-affinity AVR-PikD binding from the weaker ancestral state. In both cases the HMA domain only recently acquired the capacity to bind the AVR-PikD effector with high affinity, indicating that for most of its evolutionary history the HMA was not subject to selective pressure imposed by this blast effector. In addition, although Pikp-1 and Pikm-1 receptors evolved to produce similar phenotypic outcomes, they underwent different evolutionary trajectories to do so. These findings indicate that the evolution of NLR receptors is even more dynamic than previously thought.

Epistasis, the spice of life: Lessons from the study of the plant immune system

D. WEIGEL, Max Planck Institute for Developmental Biology, Tuebingen, Germany

My group is addressing fundamental questions in evolutionary biology, using both genome- and phenotype-first approaches. A few years ago, we discovered that Arabidopsis thaliana is a great model for the study of hybrid necrosis. This widespread syndrome of hybrid failure in plants is caused by plant paranoia - regardless of the presence of enemies, plants "think" they are being attacked by pathogens. Over the past decade, we have studied in detail the underlying genetics, finding that often one or two loci encoding NLR immune receptors are causal. NLRs make up the most variable gene family in plants, and it is not surprising that they are often involved in genome-genome conflicts. Hybrid necrosis results when NLR genes meet that have not been co-adapted. I will describe how our unique lens for the plant immune system has led to insights that were not obtained with conventional laboratory genetics.

Our goal for the next decade is to understand the genomic and geographic patterns of immune system diversity. Last year, we initiated a project, Pathodopsis in which we aim to describe genetic diversity in the host and two important pathogens, the generalist Pseudomonas and the specialist Hyaloperonospora arabidopsidis. The long-term vision is to produce maps of resistance alleles in the host, and of effector alleles in the pathogens, in order to learn who normally wins in a wild plant pathosystem - the host or the pathogen.

Arbuscular cell invasion coincides with extracellular vesicles and membrane tubules R. ROTH (1), S. Hillmer (2), C. Funaya (2), M. Chiapello (3), K. Schumacher (4), L. Lo Presti (5), R. Kahmann (6), U. Paszkowski (1), (1) University of Cambridge, U.K.; (2) EMCF, University of Heidelberg, Heidelberg, Germany; (3) Institute for Sustainable Plant Protection, 
National Research Council (IPSP-CNR), Turin, Italy; (4) COS, University of Heidelberg, Heidelberg, Germany; (5) Max Planck Institute For Terrestrial Microbiology, Marburg, Germany; (6) Max Planck Institute for Terrestrial Microbiology, Marburg, Germany

A hallmark of arbuscular mycorrhizal symbiosis (AMS) is a programmed incursion of fungal hyphae into root cortical cells to form fascinating, ephemeral tree-shaped feeding structures, called arbuscules. Within arbusculated cells symbiotic exchange of photosynthates for soil minerals occur across a shared symbiotic interstitial apoplastic matrix wedged between host and fungal membranes. Arbuscule formation and collapse coincide with a rapid turnover of plant and fungal membranes whose spatiotemporal dynamics directly influence nutrient exchange. Using 3-D TEM tomography we uncovered unexpected ultrastructural details of host and fungal membrane surfaces and the apoplastic matrix during growth and senescence of Rhizophagus irregularis arbuscules in rice ${ }^{1}$. Arbuscular hyphae were associated with abundant fungal membrane tubules (memtubs), which were also present in the phylogenetically distant AM fungus, Gigaspora rosea and invasive hyphae of the fungal maize pathogen, Ustilago maydis. Thus, structural commonalities in hyphal growth, independent of the mutualistic or parasitic outcome was revealed. In addition, our TEM analysis also showed presence of extracellular vesicles (EVs) consisting of exosomes and microvesicles in the symbiotic interstitial apoplastic matrix, providing a first indication that EVs may contribute to interkingdom communication during the intimate symbiotic association between plants and AM fungi.

Molecular dissection of the influence of humidity on bacterial virulence and plant immunity

Y. Hu, M. Yuan, J. Wu, L. Yao, S. Wan, X. XIN, Institute of Plant Physiology \& Ecology, Chinese Academy of Sciences, Shanghai, China

Despite tremendous efforts in breeding and pesticide input, plant diseases caused by pathogenic microbes represent one of the most important threats to global food security. One important reason is that we are far from understanding the basic disease susceptibility mechanisms and the molecular basis of the influences of environmental conditions, such as humidity and temperature, on plant diseases. Using the Arabidopsis thaliana-Pseudomonas syringae pathosystem, we discovered that bacterial pathogens such as $P$. syringae exploit type III effector proteins such as HopM1 and AvrE to drive the formation of "water soaking" in the leaf intercellular space (apoplast) of infected plants as a key virulence mechanism. Interestingly, "water soaking" formation was found to require high humidity, an environmental condition that is long-known to promote numerous plant diseases in the field, thereby providing a critical insight into the humidity effect on pathogen virulence. Our ongoing research demonstrates the involvement of other bacterial and plant genes in the process of "water soaking" formation. Intriguingly, we recently found that high humidity also significantly affects plant immune signaling, especially salicylic acid and jasmonic acid pathways, in Arabidopsis and rice plants. Collectively, our research begins to gain molecular insight into an important, but historically understudied aspect of the multidimensional "plant-pathogen-environment" interactions.

Low-Tech, Hi-Tech... PsyTEC? A systems-level probe of the plant-pathogen interface. D. DESVEAUX (1,2), B. Laflamme (2), M. M. Dillon (2), A. Martel (2), R. N. Almeida (2), D. S. Guttman (1,2), (1) Centre for the Analysis of Genome Evolution and Function, Canada; (2) University of Toronto, Toronto, ON, Canada

The type III secretion system is a crucial virulence apparatus of the plant pathogen Pseudomonas syringae. The effectors delivered by this system can either promote pathogenesis on susceptible host plants or activate an effector-triggered immune response on resistant plants. $P$. syringae is subdivided into host-restricted isolates termed pathovars, which each contain a unique repertoire of type III effectors that contribute to host specificity. Using the pan-genome of $\sim 500$ sequenced pathovars, we have generated a compendium of type III effectors (PsyTEC) that captures the sequence diversity of the $P$. syringae effectorome. PsyTEC consists of just over 70 families of type III effectors that can be subdivided into 529 subgroups. Functional characterization of PsyTEC is revealing its value as a powerful resource for dissecting crucial facets of plant-microbe interactions.

From mutation to population extinction: The butterfly effect of resistance breakdown in the poplar rust fungus Melampsora laricipopulina

A. PERSOONS, F. Halkett, S. Duplessis, P. Frey, S. De Mita, INRA, Champenoux, France

Following the Red Queen co-evolutionary dynamics, the arms race in a host-parasite system can lead to population extinction. Such an extinction has been described in the poplar-M. larici-populina (poplar rust fungus) pathosystem: a major adaptive event occurred in 1994 with the breakdown of the R7 resistance carried by poplar cultivars widely planted in Western Europe. This resistance breakdown resulted in the emergence and expansion of a genetic group (virulent 7) that replaced the initial avirulent 7 group which predominated previously (Persoons et al, 2017).

To understand the genetic basis and the demographic consequences of such an event, we developed a model of the demographic history of $M$. larici-populina. We selected 79 isolates spanning the date of detection of R7 breakdown, and we detected polymorphisms using whole-genome resequencing. We modelled the ancestry of these genetic groups using Approximate Bayesian Computation, which enabled us to test alternative hypotheses concerning the origin of the virulent 7 population and to estimate key demographic parameters. We used a genome scan approach based on a combination of standard neutrality tests and identified a genomic region showing signatures of a selective sweep, later confirmed by a GWAS approach as the potential location of the avirulence 7 locus. The current acquisition of a new reference genome from an avirulent 7 isolate will help characterizing the genetic basis that led to the virulence 7 emergence.

\section{Mapping barley genes shaping the rhizosphere bacteria}

C. M. ESCUDERO-MARTINEZ (1), R. Alegria-Terrazas (1), M. Maver (2), L. Pietrangelo (3), S. Robertson (1), R. Sharma (1), D. Bulgarelli (1), (1) Plant Sciences, School of Life Sciences, University of Dundee, Dundee, U.K.; (2) Faculty of Science and Technology, University of Bolzano, Bolzano, Italy; (3) Department of Biosciences and Territory, University of Molise, Campobasso, Italy

Defining the plant genetic determinants of the rhizosphere microbiota will impact on both basic science and translational agriculture. This is particularly relevant for the microbiota associated to crop wild relatives which may represent an untapped resource of beneficial functions. We 
previously demonstrated that wild and domesticated barley genotypes host a contrasting microbiota and we hypothesized that these differences are modulated, at least in part, by the plant genome. Our overarching aim is to dissect the barley genetic source of these differences, and test whether the wild-associated microbiota confers any improvement for barley growth. We used microbiota sequencing information as a 'quantitatve phenotype' in genetic mapping effort using a segreganting population between a domesticated and wild parent. This effort, conducted at a depth of thousands of SNPs in the barley genome, yielded the identification of single locus on chromosome $3 \mathrm{H}$ associated with the recruitment of taxonomically distinct bacteria, possibly representing a major regulator of plant-microbiota interactions. Strikingly, microbial profiles of barley near isogenic lines with contrasting haplotypes in the region of interest confirmed, at least in part, the role of locus $3 \mathrm{H}$ in shaping the rhizosphere microbiota. Here I present the latest results in this line of investigation.

\section{Microbe-associated molecular patterns and endophytic microbiome assembly in Arabidopsis thaliana}

C. OLDSTONE-JACKSON, F. Huang, J. Bergelson, University of Chicago, Chicago, IL, U.S.A

The plant microbiome moderates many important plant traits and is furthermore known to be shaped at least in part by the host. To efficiently engineer the microbiome to achieve desirable host phentoypes, we first must understand the factors governing microbial community assembly. The plant immune system is likely to be a key factor that determines microbial community composition. One important component of plant immunity is a suite of pattern recognition receptors that recognize microbe-associated molecular patterns (MAMPs). Since MAMPs trigger an antimicrobial response and are found across diverse microbial taxa, plant MAMP receptors may play an important role in structuring the microbiome. However, this remains untested. To determine the importance of MAMP receptors in shaping the endophytic microbiome, we grew several A. thaliana MAMP receptor knockout lines in field conditions. We then used marker gene sequencing to characterize the endophytic microbiomes of all major tissue types. The mutant plant microbiomes communities were compared to wild-type communities using a number of metrics. This research will determine if these broadly reactive immune receptors substantially influence microbiome assembly. It will also enhance our understanding the selective pressures on MAMP receptor genes and provide valuable insights for plant microbiome engineering.

Inter-organellar communication during innate immunity

Savithramma Dinesh-Kumar ${ }^{1}$, Nathan Meier ${ }^{1}$, Alexander Nedo ${ }^{2}$, Chandra Kambhamettu', Eunsook Park ${ }^{4,5}$ and Jeffrey Caplan ${ }^{2}$

${ }^{1}$ Department of Plant Biology and The Genome Center, College of Biological Sciences, University of California, Davis, CA 95616. ${ }^{2}$ Department of Biological Sciences, Delaware Biotechnology Institute, University of Delaware, Newark, DE 19711. ${ }^{3}$ Department of Computer and Information Sciences, College of Engineering, University of Delaware, Newark, DE 19711. ${ }^{4}$ Department of Plant Science, Seoul National University, Seoul, Korea, Republic of (South). ${ }^{5}$ Plant Immunity Research Center, Seoul, Korea, Republic of (South) ${ }^{5}$

The innate immune system of both plants and animals employs cell-surface and intracellular receptors to detect pathogens and trigger defenses. Emerging evidence suggests that chloroplasts play an important function during innate immunity and they also have a central role in the production of immune signals. Our recent findings demonstrated that chloroplasts dynamically change their morphology by sending out stromafilled tubular projections known as stromules during immune responses. We will discuss these findings and our recent findings on the dynamics of stromule formation and the role of cytoskeleton on stromule-driven movement of chloroplasts to nuclei during plant innate immunity.

Exo70B2 Subunit of the Exocyst Links Immune Signaling to the Secretory Pathway and Autophagy M. TRUJILLO, Albert-Ludwigs-University Freiburg, Freiburg, Germany

The exocyst is a conserved hetero-octameric complex that mediates early tethering of post-Golgi vesicles during exocytosis. Its Exo70 subunit functions as a spatiotemporal regulator by mediating numerous interactions with proteins and lipids. However, a molecular understanding of the exocyst functions, as well as the interplay between immune signaling and the secretory pathway, remains challenging. We have previously shown that Exo70B2 is required for full activation of the PAMP-triggered immunity and regulated via ubiquitination mediated by the E3 ligase PUB22. We additionally discovered that Exo70B2 interacts with and is phosphorylated by the MITOGEN-ACTIVATED PROTEIN KINASE 3 (MPK3). Identified phosphorylation sites control association to the plasma membrane at sites of high secretory activity. Moreover, we show that Exo70B2 is autophagocytosed and phosphorylation also controls its association with AUTOPHAGY-RELATED PROTEIN 8 (ATG8) via two ATG8interacting motifs. Lines expressing phosphonull variants displayed higher effector-triggered immunity and were hypersensitive to BTH, conditions known to induce the secretory pathway. Together, by uncovering the regulatory mechanism governing Exo70B2, we expose its function as a convergence point at which immune signaling, ubiquitination and autophagy meet the secretory pathway.

Insights Behind the Molecular Mechanism of Cytokinin-Induced Priming K. MCINTYRE, D. R. Bush, C. T. Argueso, Colorado State University, Fort Collins, CO, U.S.A.

Priming is the indirect enhancement of the immune response of plants to pathogens. Compared to unprimed plants, the immune response from primed plants, upon pathogen attack, is much stronger. Recent research in Arabidopsis thaliana has shown that the plant hormone cytokinin has a priming effect against biotrophic pathogens, a phenomenon we call cytokinin-induced priming. Our research demonstrates, that like other priming agents, priming with cytokinin induces a low level of defense gene expression but after a cytokinin-primed plant is challenged by a pathogen, defense gene expression is more robust. The molecular mechanisms behind priming remains largely unknown, although recent studies have indicated that chromatin modifications may play a role. Our research on chromatin mapping using Assay for Transposase-Accessible Chromatin using sequencing (ATAC-Seq) indicates that priming by cytokinin involves differential accessibility to various regions in the genome. Further, we show that priming by cytokinin does little to alter the plant metabolome initially. However, after the plant experiences pathogen challenge, the metabolic profile in a cytokinin-primed plant is significantly distinct from unprimed plants. We propose that cytokinin-induced chromatin regulation and alteration of the metabolomic profile provide insights into the general mechanisms of defense priming against biotic stress. 
Cross-Kingdom small RNA trafficking between plants and fungal pathogens

Qiang Cai, Baoye He, Shumei Wang and Hailing Jin*

Department of Microbiology \& Plant Pathology, University of California, Riverside, CA 92521, U.S.A.

Small RNAs (sRNAs) are a class of short non-coding RNAs that mediate gene silencing in a sequence-specific manner. We have demonstrated that some sRNAs from eU.K.aryotic pathogens, such as Botrytis cinerea, the fungal pathogen that causes grey mold disease on more than 1000 plant species, can be transported into host plant cells and suppress host immunity genes for successful infection. We recently discovered that such cross-kingdom RNAi is bi-directional. Plants can also send small RNAs into pathogens using extracellular vesicles to silence its virulence genes as part of its immune responses. During the co-evolutionary arms race with the pathogen, plants have adapted exosome-like extracellular vesicles as one of the major pathways to deliver sRNAs into fungal cells and induce cross-kingdom RNAi. The potential mechanisms of sRNA loading into the plant extracellular vesicles will be discussed.

Furthermore, we also discovered that $B$. cinerea can take up double-stranded RNAs and sRNAs from the environment. Applying sRNAs or dsRNAs that target fungal genes on the surface of fruits, vegetables and flowers significantly inhibits grey mold disease. Such pathogen genetargeting RNAs represent a new generation of fungicides that are durable and eco-friendly.

Escape from the battlefront: How Colletotrichum higginsianum uses plasmodesmata to establish an infection M. OHTSU, J. Jennings, M. Johnston, X. Liu, C. Faulkner, John Innes Centre, U.K.

Cell-to-cell communication is essential for multicellular organisms. In plants, plasmodesmata (PD) play a key role in cell-to-cell communication. Almost every plant cell is connected to its neighbours via PD. PD allow the movement of various molecules from small metabolites to large proteins and mRNAs between cells, thus PD connect cells to form a "highway" by which symplastic signals spread. Interestingly, some plant pathogens also use PD as an effective pathway for intercellular hyphal passage and for the spread virulence factors in plant tissues. We have observed that the regulation of PD is critical for PAMP responses and that pathogens can supress this during their infection process. These results indicate that cell-to-cell communication via PD are a battlefront between plants and pathogens. In this study, we aim to identify effectors of Colletotrichum higginsianum which target symplastic communication via PD to elucidate the mechanisms involved in how pathogens manipulate and exploit PD. We used genome and transcript sequence data to select effector candidates, and then we established a live-cell imaging screen to determine their localisation and mobility. From this screen, we have identified effector candidates that have high cell-to-cell mobility and others that localise to PD. These effectors are candidates which could both regulate cell-to-cell communication via PD and exploit them to access distal cells and tissues.

\section{An H3K27me3 demethylase-HSFA2 loop controls thermomemory associated with attenuated immunity and early flowering in Arabidopsis \\ Z. HE, Institute of Plant Physiology \& Ecology, SIBS, CAS, Shanghai, China}

Global warming has profound effects on plant growth and fitness. Plants have evolved sophisticated epigenetic machinery to respond quickly to heat and exhibit transgenerational memory of the heat-induced release of post-transcriptional gene silencing (PTGS). However, how the thermomemory is transmitted to progeny and the physiological relevance are elusive. Here we show that heat-induced HEAT SHOCK TRANSCRIPTION FACTOR A2 (HSFA2) directly activates the H3K27me3 demethylase RELATIVE OF EARLY FLOWERING 6 (REF6), which in turn derepresses HSFA2. REF6 and HSFA2 establish a heritable feedback loop, and activate an E3 ubiquitin ligase, SUPPRESSOR OF GENE SILENCING 3 (SGS3)-INTERACTING PROTEIN 1 (SGIP1). SGIP1-mediated SGS3 degradation leads to inhibited biosynthesis of trans-acting siRNA (tasiRNA). The REF6-HSFA2 loop and reduced tasiRNA converge to release HEAT-INDUCED TAS1 TARGET 5 (HTT5), which drives early flowering but attenuates immunity. Thus, heat induces transmitted phenotypes via a coordinated epigenetic network involving histone demethylases, transcription factors, and tasiRNAs, ensuring reproductive success and transgenerational stress adaptation.

Movement and propagation of Candidatus Liberibacter asiaticus in its plant host and insect vector D. Achor (1), M. Ghanim (2), S. Ben-Mahmoud (3), S. Y. Folimonova (1), S. Gowda (1), A. LEVY (4), (1) University of Florida, U.S.A.; (2) Volcani Center, Rishon LeZion, Israel; (3) University of California, Davis, U.S.A.; (4) University of Florida, FL, U.S.A.

Citrus greening is caused by the phloem-restricted bacteria Candidatus Liberibacter asiaticus (CLas) and is transmitted by the Asian citrus psyllid Diaphorina citri. CLas is an intracellular bacterium, but very little is known about its interactions with the plant or insect at the cellular level. Here we used microscopy and gene expression analyses to study CLas movement and host responses inside the plant and vector. We show that CLas enters $D$. citri gut cells by endocytosis and that it forms a large replicative vacuole inside the insect's gut and reproductive organs. The formation of these vacuoles is accompanied by dramatic re-organization of the endoplasmic reticulum (ER) that associate with these intracellular bodies. In the plant, CLas propagates in the cytoplasm of sieve elements, and movement is carried out by its elongated form through the phloem pores. The phloem responds to the bacterial presence by callose and phloem-protein upregulation that seals the pores. However, the levels and characteristics of this respond depend on the specific plant tissue examined. Our results show that in its insect vector, CLas modifies the host endomembrane system to move and create an isolated safe environment to support its own propagation. In the plant, host response is differential, and depends on the specific plant tissue. Understanding the molecular interactions that are involved in these pathogen-host processes may open new opportunities for controlling CLas.

The structure of the rice leaf microbiome is controlled by a complex host-microbe interplay V. Roman-Reyna (1), P. Dale (2), I. L. Quibod (2), A. Nicolai (2), G. C. Simon (3), R. Mauleon (2), R. OLIVA (4), (1) Ohio State University, U.S.A.; (2) International Rice Research Institute, Philippines; (3) New York University, U.S.A.; (4) International Rice Research Institute, Los Banos, Philippines 
Microbial communities that live in association with plants carry a great diversity of metabolic capabilities. The composition of these communities is usually influenced by host factors but also by complex microbe-microbe interactions. We characterized the composition, structure, and functional profile of the rice leaf microbiome using a global panel of 3,024 accessions grown in two major rice-producing areas, China and the Philippines. Diversity and abundance appeared to be highly influenced by environment and rice varietal group. Despite significant differences in diversity, co-occurrence network analysis identified three microbial hubs that define the network. Moreover, the structure and metabolic profiles of the community in China and the Philippines appear to be conserved, suggesting that core functions rather than individual members define the consortia composition. Interestingly, genome-wide associations identified rice genomic region controlling the abundance of the hubs. These signals often co-localize with genes related to immunity and stress responses suggesting a strong contribution of the host in shaping the leaf microbiome structure. Analysis of the apoplastic microbiome of near-isogenic lines suggests that different immunity component have an impact on specific microbial groups. Our data provide insight into the factors influencing microbial assemblage but also opens the door for future initiatives to modulate rice consortia for crop improvement efforts.

Plant immunity regulated by cell wall integrity: Unveiling novel carbohydrate-based molecular patterns

H. Mélida (1), L. Bacete (1), D. Rebaque (2), G. López (1), E. Miedes (1), L. Jorda (1), A. MOLINA (1), (1) Centro de Biotecnología y Genómica de Plantas, Universidad Politécnica de Madrid, Pozuelo de Alarcón, Spain; (2) PlantResponse Biotech, Pozuelo de Alarcón, Spain

Plant innate immune system can be activated by microbe-associated molecular patterns (MAMPs), but also by damage-associated molecular patterns (DAMPs) that trigger immune responses upon recognition by plant Pattern Recognition Receptors (PRRs). DAMPs are signaling molecules released from host cellular structures, like plant cell walls, upon pathogen infection or wounding. Despite the hypothesized important role of DAMPs in plant-pathogen interactions, a very limited number of DAMPs are well characterized. Typical examples of carbohydrate-based DAMP/MAMPs are oligogalacturonides, $\beta$-glucans and chitin. Cell wall extracts from Arabidopsis thaliana cell wall mutants showing altered disease resistance to several pathogens were chemically and chromatographically fractionated and these fractions were tested for their capacity to activate the immune system. Notably, several wall fractions were found to trigger immune responses and those showing the highest activity were further characterized. These analyses pointed to a role of pentose oligosaccharides in triggering immunity. Further analyses revealed that $\beta-1,4-$ xylooligosaccharides of specific degrees of polymerization carrying arabinose decorations are sensed as DAMPs by plants. The molecular mechanisms regulated by these and additional novel cell wall-derived DAMPs in plant immunity are being characterized and will be presented. The potential differences between DAMP and MAMP-triggered immunity will be also discussed.

Evidence for host specificity within root-associated bacterial communities

K. WIPPEL (1), K. Tao (2), R. Zgadzaj (1), S. Radutoiu (3), P. Schulze-Lefert (1), R. Garrido-Oter (1), (1) Max Planck Institute for Plant Breeding Research, Cologne, Germany; (2) University of Aarhus, Denmark; (3) University of Aarhus, Aarhus, Denmark

In nature, different plant species are colonized by distinct microbiotas. Thus, while the composition of root-associated bacterial consortia is similar at higher taxonomic ranks across species, it is highly variable at lower levels, even in plants grown in the same soil. The factors involved as well as the role of the host in driving these differences are currently unknown. To investigate this, we have performed colonizationcompetition experiments using the phylogenetically distant plant species Arabidopsis thaliana and Lotus japonicus. We established an extensive whole genome-sequenced bacterial culture collection of roots from Lotus grown in soil previously used for a collection from Arabidopsis. This allowed us to design a synthetic community (SynCom) consisting of strains from both hosts covering families of the core microbiota, which was used as inoculum. Using two fully independent SynComs, we found signatures of host specificity, i.e., strains exhibited higher overall abundance on their respective original host than on the other host. To decipher the mechanism underlying this preferential host association, we are employing an array of plant mutants compromised in either plant innate immunity or secondary metabolism, as well as multiple alternative SynComs. This work extends our knowledge about processes driving host microbiota specificity and evolution and provides new perspectives for tailored design of stable and beneficial microbial inoculants.

Revealing the "box" code: The spatial and temporal regulation of plant-parasitic nematode pathogenicity C. PELlEGRIN (1), H. Beasley (1), B. Mendy (2), M. S. Hasan (2), D. Chopra (2), C. Hiltl (2), H. Chen (2), V. Cevik (2), O. Chitambo (2), F. M. W. Grundler (2), S. Siddique (3), S. Eves-van den Akker (1), (1) University of Cambridge, U.K.; (2) Rheinische Friedrich-WilhelmsUniversity of Bonn, INRES Molecular Phytomedicine, Germany; (3) Department of Entomology and Nematology, University of California, CA, U.S.A.

Plant-parasitic nematodes are an important group of plant pathogens that threaten current and future food security. Among them, the cyst nematodes parasitize some of the most important crop species. To cause disease, cyst nematodes inject effector proteins into the plant. Nematode effectors are produced primarily in either the dorsal or sub-ventral glands. Hundreds of dorsal gland effectors are unified by a six base-pair noncoding DNA motif, termed the "DOG box". In a recent effort, a transcription factor that can recognize the DOG-box was identified: a candidate "DOG box reader". To understand the regulation of plant-parasitic nematode parasitism, the key questions that remain are: what are the genetic signatures and readers of different gland cells and different times of infection, and to what extent are these regulatory mechanisms conserved between cyst nematodes? We have used gene network analysis of three different cyst nematodes species to identify co-expressed genes associated with key steps of the infection cycle, and a series of candidate master regulators that lie upstream. Functional analysis of these master regulators associated with genome-wide expression analysis of both gland cells has begun to reveal the spatio-temporal regulatory landscape that sustains the pathogenicity of these devastating pests.

Pattern recognition receptor complexes confer direct apoplastic resistance against bacterial water acquisition in plants T. Hirase (1), S. YASUDA (1), L. Verdoucq (2), K. Yamada (3), I. Finkemeier (3,4), H. Nakagami (3,5), S. Y. He (6), C. Maurel (2), Y. Saijo (1), (1) Nara Institute of Science and Technology, Japan; (2) Centre national de la recherche scientifique, France; (3) Max Planck Institute for Plant Breeding Research, Germany; (4) University of Münster, Germany; (5) RIKEN Center for Sustainable Resource Science, Japan; (6) Michigan State University, U.S.A. 
Plant-pathogen interactions are profoundly influenced by water conditions, and often result in severe plant diseases under high humidity. The bacterial pathogen Pseudomonas syringae pv. tomato (Pst) DC3000 develops an aqueous leaf apoplast with type III secretion effectors (T3Es) for virulence promotion. However, whether and if so how plants directly resist against bacterial water acquisition is poorly understood. Here, we report a critical role for the plasma membrane intrinsic proteins (PIPs) in pattern triggered immunity to bacteria. In Arabidopsis thaliana, PIPs physically associate with the pattern recognition receptors (PRRs) FLS2 and PEPR1 for bacterial flagellin and endogenous Pep peptides, respectively. Remarkably, of the thirteen PIP members, dysfunction of one member permits an effectorless Pst strain to cause apoplastic water accumulation and extensive proliferation. Genetic and biochemical evidence indicates that PIP phosphorylation in response to PRR activation leads to direct resistance against bacterial water soaking, possibly via enhanced water transport, and that the PRR-associated kinase BAK1 can mediate this PIP phosphorylation event. Conversely, to promote water soaking, Pst employs specific T3Es to intercept this novel PRR signaling output. Our findings suggest that PIP phosphorylation by PRR complexes provides a basis for apoplastic water control during bacterial resistance.

\section{A Phytophthora Effector Suppresses Host-induced Gene Silencing Mediated by Secondary Small RNAs}

Y. Hou (1), Y. Zhai (1), W. Ye (2), L. Feng (3), H. Zand Karimi (4), B. D. Rutter (4), R. W. Innes (4), J. Ma (5), J. Zhai (3), W. MA (6), (1) University of California, Riverside, CA, U.S.A.; (2) Nanjing Agricultural University, China; (3) Southern University of Science and Technology, China; (4) Indiana University, Bloomington, IN, U.S.A.; (5) Fudan University, Shanghai, China; (6) University of California, Riverside, Riverside, CA, U.S.A.

Plants have evolved a complex immune system that must be defeated by pathogens to establish infection. We discovered that Phytophthora infection of Arabidopsis leads to increased accumulation of a diverse pool of secondary small interfering RNAs (siRNAs) derived from specific gene transcripts. Instead of regulating endogenous genes, these siRNAs are found in extracellular vesicles and silence target genes in Phytophthora during natural infection. Introduction of plant siRNAs in Phytophthora leads to developmental deficiency and abolished virulence, while Arabidopsis mutants defective in the biogenesis of these siRNAs are hypersusceptible. Intriguingly, $\underline{P}$ hytophthora encodes suppressors of RNA silencing (PSRs), which facilitate the infection of plant hosts. In particular, the effector PSR2 specifically inhibits the secondary siRNA pathway in Arabidopsis and promotes infection. These findings reveal siRNAs as antimicrobial agents targeting pathogens through host-induced gene silencing and highlight an arms race between plant defense mechanisms and pathogen effectors. We will discuss recent progress on the functional and structural characterization of PSR2, through which a conserved module that is prevalent in Phytophthora effectors was identified. Detailed analysis on the siRNA-generating transcripts that contribute to trans-kingdom RNAi will also be reported.

Dynamic ubiquitination determines NPR1 transcriptional coactivator activity M. J. SKELLY (1), J. J. Furniss (1), H. L. Grey (1), K. W. Wong (1), S. H. Spoel (2), (1) University of Edinburgh, Edinburgh, U.K.; (2) School of Biological Sciences, University of Edinburgh, Edinburgh, U.K.

Activation of systemic acquired resistance is associated with transcriptome reprogramming induced by the unstable coactivator NPR1. Immuneinduced ubiquitination and proteasomal degradation of NPR1 are thought to facilitate continuous delivery of active NPR1 to target promoters, thereby maximising gene expression. Because of this potentially costly sacrificial process, we investigated if ubiquitination of NPR 1 plays transcriptional roles prior to its proteasomal turnover. Our data suggests that ubiquitination of NPR1 is a processive mechanism in which initial modification by a Cullin-RING E3 ligase promotes its transcriptional activity. Only when polyubiquitination of NPR1 is enhanced by the E4 ligase, UBE4, it is targeted for proteasomal degradation. Furthermore, ubiquitin chain extension activities are opposed by UBP6/7, two proteasome-associated deubiquitinases that stabilise NPR1 and promote its transcriptional activity. Our findings suggest that the sequential actions of E3 and E4 ligases, balanced by opposing deubiquitinases fine-tune NPR1 transcriptional activity without strict requirement for sacrificial turnover. This likely serves as a regulatory mechanism to ensure that the transcriptional window of opportunity for NPR1 to activate target genes remains flexible, allowing adjustment according to immune demand.

CAMTA1: A novel regulator of nodulation

A. YAMAZAKI (1), K. Toyooka (1), N. Takeda (2), A. Akamatsu (3), A. Miyahara (4), M. Nagae (4), Y. Umehara (4), M. Hayashi (1), (1) RIKEN, Japan; (2) Kwansei gakuin university, Japan; (3) KGU, Japan; (4) NIAS, Japan

Legumes are able to fix atmospheric nitrogen in the nodules that are generated as a consequence of an intimate interaction between hosts and symbiotic rhizobia. Intensive molecular investigations have revealed genes that are indispensable or important for root nodule symbiosis (RNS), however, negative regulations that fine-tune RNS have yet unclear. Here, we performed a suppressor screening in the nodulation-defective castor mutant and identified a calmodulin-binding transcription activator1 (CAMTA1) as a novel inhibitor of RNS. A loss-of-function mutation in CAMTA1 enabled castor to form infected nodules upon rhizobial inoculation, which was not accompanied by the induction of the symbiotic calcium oscillation called calcium spiking or the formation of infection threads (ITs) in root hairs. The bacteroid development in the infected cells and the IT formation inside nodules looked intact in the castor camtal double mutant. A genetic epistasis and protein-protein interaction assays indicated CYCLOPS, a transcription factor required for the plant-microbe symbioses, as a potential target of CAMTA1 during RNS. In addition, the activation of a tandem CYCLOPS-responsive element combined with a NIN (Nodule Inception) minimal promoter by gain-of-function CCaMK and CYCLOPS was attenuated by the presence of CAMTA1 in tobacco. Our data suggested that CAMTA1 negatively affected nodule organogenesis by interfering with the activation of CYCLOPS by CCaMK.

How PRR signaling regulates immunity

Michaela Kopischke ${ }^{1,2}$, Yi Liu ${ }^{1}$, Timothy Hawkins ${ }^{3}$, Tobias Maierhofer ${ }^{4}$, Jan Sklenar ${ }^{2}$, Frank Menke², Dietmar Geiger ${ }^{4}$, Patrick Hussey ${ }^{3}$, Rainer Hedrich $^{4}$, Silke Robatzek ${ }^{1,2}$

${ }^{1}$ Biocenter, LMU München, DE; ${ }^{2}$ The Sainsbury Laboratory, Norwich Research Park, U.K.; ${ }^{3}$ University of Durham, U.K.; ${ }^{4}$ Biocenter, University of Würzburg, DE 
Our interest is to understand how plants defend themselves against infection and how successful pathogens overcome plant immunity. Perception of microbe-associated molecular patterns (MAMPs) by pattern recognition receptors (PRRs) results in the closure of stomata, leaf epidermal pores that represent entry gates for pathogens when open. We identified a guard cell expressed anion channel as a direct phospho-regulatory target of the PRR complex in acute MAMP-induced stomatal closure and anti-fungal immunity. Further, we show that PRR signaling, in addition to triggering acute stomatal closure, sustains the closure of stomata. The latter requires activation-dependent regulation of a FLS2-associated RAB7 GTPase and its NET4 effectors, which are actin-to-tonoplast tethers. These provide examples of acute and sustained anti-microbial immunity in plant body surface protection.

This work is supported by the Gatsby Charitable Foundation, the European Research Council (ERC), and the German Research Foundation (DFG).

Specialisation of chitin signaling pathways at plasmodesmata

C. Cheval, M. Johnston, S. Samwald, C. FAULKNER, John Innes Centre, U.K.

The plant immune system is characterised as a cell autonomous response system; in general, all plant cells are capable of pathogen perception and response. However, plasma membrane-lined pores that connect neighbouring cells called plasmodesmata (PD) are regulated during immune responses, identifying that cell-to-cell connectivity is a component of immune signaling. PD close in response to a range of microbe associated molecular patterns (MAMPs), mediated by specialised immune signaling components located at PD. For chitin, the LysM domain, GPI-anchored receptor protein LYM2 mediates PD closure via complex formation with an additional LysM domain receptor kinase. Upon chitin binding, this receptor complex signals via calcium and reactive oxygen species signaling that ultimately triggers callose deposition and PD closure. This is a rapid signaling event that occurs independently of other chitin-triggered responses that occur in the plasma membrane, identifying spatial resolution in receptor signaling cascades in plant cells. While plasma membrane located responses to chitin involve the same signaling molecules, the signaling cascade in the PD membrane involves different mechanisms by which these molecules are produced. We are currently using a range of imaging and biochemical approaches to determine receptor complex and signaling dynamics in the PD plasma membrane and how this underpins cell to cell connectivity and its contribution to both defence and infection.

The SYFO-mediated cell wall- plasma membrane- cytoskeleton continuum is required for symbiotic infections in Medicago truncatula P. LIANG (1), J. Keller (2), C. Schmitz (1), P. M. Delaux (2), T. Ott (3), (1) University of Freiburg, Freiburg, Germany; (2) LRSV UMR5546 CNRS/Université de Toulouse III, Castanet-Tolosan, France; (3) University of Freiburg, Germany

Plant cell infections are tightly orchestrated by cell wall (CW) alterations, plasma membrane (PM) signaling and dynamic remodelling of the cytoskeleton. During root nodule symbiosis these processes result in morpho-dynamic responses including root hair curling, PM invagination and polar growth of a tubular infection structure, the infection thread (IT). However, the molecular details driving and guiding these PM remodelling events remain to be unravelled. Here, we studied a formin protein (SYFO) from the model legume M. truncatula. Phenotypical analysis of RNAimediated knock-down and syfo mutant roots clearly indicates that the encoded protein is required for efficient rhizobial colonization of root hairs. SYFO itself creates a proteinaceous bridge between the $\mathrm{CW}$ and the polarized cytoskeleton. It binds to $\mathrm{CW}$ components via a proline-rich $\mathrm{N}-$ terminal segment and co-localizes with callose depositions during rhizobial infections. On the cytoplasmatic site of the plasma membrane SYFO is associated with actin accumulations supporting the hypothesis that it contributes to cell polarization in vivo. This is further sustained by the fact that cell shape changes can be induced in a stimulus-dependent manner in root protoplasts expressing SYFO. Taken together we provide evidence for a so far undescribed protein controlling rhizobial infections in model legume.

\section{Develop effective protectants and therapies to manage citrus HLB and potato ZC using a novel class of citrus-derived antimicrobial} peptides

C. Y. HUANG (1), D. D. Niu (2,3), G. Kund (4), M. Jones (4), K. Godfrey (5), J. Trimble (4), A. Ute (6), K. Bowman (7), C. Ramadugu (8), H. Jin (9), (1) University of California Riverside, Riverside, CA, U.S.A.; (2) Department of Plant Pathology and Microbiology, UCR, U.S.A.; (3) Nanjing Agricultural University, Nanjing, China; (4) Department of Entomology, UCR, U.S.A.; (5) Contained Research Facility, UCD, U.S.A.; (6) Southwest Florida Research \& Education Center, UF, U.S.A.; (7) US Horticultural Research Laboratory, USDA, Fort Pierce, FL, U.S.A.; (8) Department of Botany and Plant Science, UCR, U.S.A.; (9) University of California, Riverside, CA, U.S.A.

Candidatus Liberibacter species are the causal agent of devastating diseases for several crops. C. L. asiaticus (CLas) causes Huanglongbing (HLB), which is the most serious citrus disease and is vectored by Asian citrus psyllid (ACP). C. L. solanacearum (CLso), which is vectored by the potato psyllid, causes another important disease, potato zebra chip (ZC). Despite extensive efforts, there is still no efficient disease management for HLB or potato ZC. Through comparative analysis of small RNAs and mRNAs between HLB-tolerant and susceptible varieties, we identified a list of regulatory components of host natural immune responses against HLB. To functionally characterize these regulators, we developed a novel screening assay, Nicotiana benthamiana ( $\mathrm{Nb}$ )/potato psyllid/CLso interacting system, as a closely related system to HLB and potato ZC. One of the validated functional regulators is a novel class of antimicrobial peptides (AMPs), SAMP. SAMP is resistant to plant proteolysis and highly stable in high temperature. SAMP can directly kill L. crescens, the sole culturable member of the Liberibacter genus. SAMP also induces defense responses that protect plants from future CLas infection. Thus, SAMPs can be developed into not only therapeutic solutions to manage HLB positive trees by inhibiting CLas, but also protectants to vaccinate and protect young trees by stimulating plant immune systems. We further demonstrate that SAMP application can also inhibit potato ZC disease.

Functions of extracellular vesicles in bacterial infection of plants M. JANDA (1,2), K. Rybak (2,3), E. Stigliano (3,4), V. Marosi (2), A. Klingl (5), J. G. Malone (6), J. Sklenar (3), F. L. H. Menke (3), S. Robatzek (2,3), (1) University of Chemistry and Technology Prague, Prague, Czech Republic; (2) Ludwig-Maximilians-Universität München, Genetics, Martinsried, Germany; (3) The Sainsbury Laboratory, University of East Anglia, Norwich Research Park, Norwich, U.K.; (4) The John Innes Centre (JIC), Norwich Research Park, Norwich, U.K.; (5) Ludwig-Maximilians-Universität München, Botany, Martinsried, Germany; (6) John Innes Centre (JIC), Norwich Research Park, Norwich, U.K. 
Extracellular vesicles (EVs) define lipid bilayer-enclosed, cytosol-containing spheres and are important structures in the interaction between hosts and pathogens. Yet, their role in the infection of plants by disease-causing bacteria is not understood. We recently found that Pseudomonas syringae pv. tomato (Pto) DC3000 releases EVs, characterized by electron microscopy (SEM, TEM), Dynamic light scattering (DLS) and nanoparticles tracking analysis (NTA). Although application of purified Pto EVs induced prototypic pattern triggered immune (PTI) responses such as oxidative burst, defence gene expression and seedling growth inhibition, the vesicles promoted in planta growth of the bacterium. Consistently, EVs suppressed the flagellin (flg22)-induced oxidative burst. Using proteomics, we determined the Pto EV proteome. Interestingly, a large proportion of the EV proteins appears to be regulated in response to PTI. In planta expression of selected EV cargo modulated the flg22induced oxidative burst, suggesting that these bacterial proteins interfere with the plant's immune system. We will discuss possible modes of EV protein delivery and function. In conclusion, we have characterized the EV proteome of a plant pathogenic bacterium and their impact on the interaction of Pto with its host plant.

Molecular determinants of symbiosis or defense identified in the model legume Lotus japonicus S. RADUTOIU (1), Z. Bozsoki (1), S. B. Hansen (2), K. Gysel (2), D. Lironi (2), F. Feng (3), G. Oldroyd (3), J. Stougaard (1), K. R. Andersen (2), (1) University of Aarhus, Aarhus, Denmark; (2) University of Aarhus, Denmark; (3) Sainsbury Laboratory Cambridge University, Cambridge, U.K.

Chitin oligomers derived from fungal cell walls are recognized as PAMPSs and elicit defense responses in plants. In contrast, symbiotic nitrogenfixing rhizobia bacteria produce Nod factors, species-specific decorated chitin molecules, that are crucial for initiation of symbiosis. Plant receptors have been shown to be essential for perception of chitinous molecules. Rice CEBiP and Arabidopsis CERK1 are indispensable for chitin-elicited defense signaling, while in Lotus japonicus NFR1 and NFR5 are required for Nod factor perception and signaling. Legumes have the capacity to recognize both types of chitinous molecules and induce distinctive responses: immunity or symbiosis. Chitin and Nod factor receptors share a high level of similarity in their amino acid sequences, are expressed in the same type of root cells, yet drive signaling pathways leading to opposing outcomes. We have used a combination of plant genetics, functional characterization, biochemical investigations and structural biology to determine the molecular mechanism enabling these distinct responses.

Genomic characterisation of the Alternaria alternata species group informs diagnostics for host specific pathotypes A. D. ARMiTAGE (1), H. M. Cockerton (1), S. Sreenivasaprasad (2), J. Woodhall (3), C. Lane (4), C. Lane (4), R. J. Harrison (1), J. P. Clarkson (5), (1) NIAB EMR, East Malling, U.K.; (2) University of Bedfordshire, Luton, U.K.; (3) University of Idaho, Parma, ID, U.S.A.; (4) Fera Science Ltd, York, U.K.; (5) University of Warwick, U.K.

The Alternaria alternata species group (Alternaria sect. alternaria) represents a diverse group of saprophytes, human allergens and plant pathogens. Increased movement of plant material risks establishment of host specific pathotypes in Europe. Alternaria taxonomy has benefited from recent phylogenetic revision, but the basis of differentiation between lineages within the group is unclear. We report reference genomes for the apple and pear pathotypes as well as draft assemblies for a further 10 isolates representing Alternaria tenuissima and Alternaria arborescens. These assemblies provide insights into differentiation of these taxa, including identification of unique effector and toxin complements. Characterisation of apple and pear conditionally dispensable chromosomes (CDCs) showed that these regions carry effectors, transcriptions factors and heterokaryon incompatibility loci in addition to their known toxin-synthesis gene clusters. Profiling a wider set of 89 isolates for mating type idiomorphs indicates that gene flow has occurred since the formation of $A$. tenuissima and A. arborescens lineages. We provide evidence that the apple pathotype is polyphyletic, which has implications for control of this EPPO A1 quarantine pathogen. We also developed primers to a gene from the apple pathotype toxin gene cluster with homologs in all tested pathotypes. These primers identify and differentiate apple, pear and strawberry pathotypes, providing new tools for pathogen diagnostics.

Bacterially secreted defense peptide StPep1 stimulates root-knot nematode resistance in potato L. Zhang, C. A. GLEASON, Washington State University, Pullman, WA, U.S.A.

Root-knot nematodes (Meloidogyne spp.) are small roundworms that infect plant roots and contribute to billions of dollars in agricultural losses each year. To control root-knot nematodes on potato, we have studied peptide defense elicitors, which are small plant peptides that stimulate plant defense responses. We have studied the potato defense elicitor StPep1 and have found that exogenous treatment of potato roots with synthetic StPep1 reduced root-knot nematode galling by $\sim 50 \%$. Peptide elicitors can be delivered by spraying or soaking the plant roots with the synthetic peptides. However, to create an alternative delivery system, we have looked at current biocontrol bacteria and their secretion systems. We transformed Bacillus subtilis to produce and secrete StPep1. The data show that the rhizobacteria can be a useful tool for defense peptide production. When potato plants were treated with the transgenic Bacillus prior to nematode inoculation, the final number of root-knot nematodes in the roots was significantly reduced. We have performed transcriptome analyses on StPep1-treated potato roots to define the mode of action of the StPep1 in triggering root resistance. Overall, we demonstrate that Pep1-secreting Bacillus triggers plant defenses and can provide enhanced immunity against an important root pathogen.

Polycomb repressive complex 2 positively regulates systemic immunity and priming in Arabidopsis thaliana Y. TAJIMA (1), M. Sato (2), E. M. Reimer-Michalski (3), E. P. I. Loo (1), B. Kracher (3), F. Turck (3), Y. Saijo (1), (1) Nara Institute of Science and Technology, Japan; (2) Hokkaido University, Japan; (3) Max Planck Institute for Plant Breeding Research, Germany

Localized defense activation at pathogen challenged leaves leads to systemic acquired resistance (SAR) at distal unchallenged leaves. During and/or after SAR, defense-related genes become sensitized to a subsequent stress, designated defense priming. Our evidence suggests a critical role for epigenetic control via histone $\mathrm{H} 3$ tri-methylation at lysine 4 (H3K4me3) and $\mathrm{H} 3 \mathrm{~K} 27 \mathrm{me} 3$ in systemic defense priming. SAR is lowered not only in atx 1 plants defective in H3K4me3-depositing trithorax protein complex, but also in clf plants defective in H3K27me3-depositing polycomb repressive complex2 (PRC2). Conditional nuclear translocation of CLF validates the requirement for PRC2 function in systemic leaves during priming. Genome-wide RNA-seq and ChIP-seq analyses reveal an inventory of priming target genes, which are overrepresented with 
genes increasing H3K4me3 without H3K27me3 depletion. Remarkably, priming defects of these target genes in clf plants are associated with a decrease in $\mathrm{H} 3 \mathrm{~K} 4 \mathrm{me} 3$ at their loci. These results suggest a positive role for PRC2 and $\mathrm{H} 3 \mathrm{~K} 4 \mathrm{me} 3$ in systemic defense priming. We are currently testing possible correlation and its functional significance between primary induction, H3K4me3 deposition and priming states in systemic leaves for the identified priming target genes.

Insect Eggs Trigger Inter-plant Systemic Acquired Resistance and Enhanced Insect Performance

Z. ORLOVSKIS, P. Reymond, University of Lausanne, Lausanne, Switzerland

Recognition of molecular patterns from plant pathogens or herbivores trigger not only responses in local plant tissue but also activate a broadspectrum plant defence priming in distal leaves against potential future attacks, leading to systemic acquired resistance (SAR). Additionally, attacked plants encode a signal that triggers SAR response in neighbouring plants lacking initial exposure to pathogen elicitors. However, the discrimination between molecular mechanisms involved in the SAR signal generation in local tissue and decoding in distal tissues or neighbouring plants are not fully understood. Here, we demonstrate that insect eggs induce not only intra-plant but also inter-plant SAR against Pseudomonas syringae via a root-mediated signal in Arabidopsis thaliana. Furthermore, egg-induced activation of SAR in neighbouring plants is coupled with increased insect larval performance on these plants. Thus, these results suggest that insects have evolved strategies to favour optimal development of future larvae by providing a niche of host plants with reduced infection and reduced anti-insect defences.

A necrotrophic effector from Parastagonospora nodorum triggers programmed cell death by targeting two distinct non-homoeologous wheat sensitivity genes

J. K. RICHARDS (1), G. K. Kariyawasam (2), Z. Liu (2), J. D. Faris (3), T. L. Friesen (3), (1) Louisiana State University Agricultural Center, Baton Rouge, LA, U.S.A.; (2) North Dakota State University, Fargo, ND, U.S.A.; (3) Cereal Crops Research Unit, USDA-ARS, Fargo, ND, U.S.A.

Parastagonospora nodorum, a necrotrophic fungal pathogen of wheat, uses an array of necrotrophic effector (NE) proteins to facilitate disease. The perception of NEs by host sensitivity proteins results in the initiation of programmed cell death (PCD). Nine NE-sensitivity gene interactions have been observed within this pathosystem, however, genes encoding SnTox2 or SnTox6 remain undiscovered. Genome sequencing of 198 P. nodorum isolates and phenotyping of host differential lines enabled an association mapping (AM) approach to identify novel NEs. AM using 322,613 polymorphisms identified a locus associated with virulence on differential lines BG223 (Snn2) and ITMI37 (Snn6) and harbored a candidate gene encoding a predicted effector. Disruption of SnTox2/6 in isolate Sn4 resulted in the loss of virulence on both Snn2+ and Snn6+ lines, as well as failure to detect Snn2 and Snn6 QTL when inoculated onto the BG and ITMI populations, respectively. Transformation of avirulent isolate Sn79-1087 with SnTox2/6 resulted in virulence on lines harboring Snn2 or Snn6, as well as detection of the aforementioned QTL. SnTox2/6 is upregulated in planta, reaching peak expression at $24 \mathrm{hr}$ post-inoculation. Putative epistatic interactions were also detected, with the deletion of SnTox2/6 affecting the efficacy of SnTox1 or SnTox3 interactions with corresponding sensitivity genes. The results indicate SnTox $2 / 6$ is a single NE that elicits PCD via detection by two non-homoeologous host targets.

The CC domains of NLR-type pathogen receptors play essential roles in oligomerization, network formation and immune signaling T. A. Wroblewski (1), L. Spiridon (2), E. C. Martin (3), A. J. Petrescu (2), K. Cavanaugh (1), M. J. Truco (4), H. Xu (5), D. Gozdowski (6), K. Pawłowski (6), R. W. Michelmore (1), F. L. TAKKEN (7), (1) University of California, Davis, CA, U.S.A.; (2) Institute of Biochemistry of the Romanian Academy, Bucharest, Romania; (3) Institute of Biochemistry of the Romanian Academy,, Bucharest, Romania; (4) UC Davis- Genome center, Davis, CA, U.S.A.; (5) The Genome Center, University of California-Davis, Davis, CA, U.S.A.; (6) Faculty of Agriculture and Biology, Warsaw University of Life Sciences, Warsaw, Poland; (7) University of Amsterdam, Amsterdam, Netherlands

Nucleotide-binding Leucine-rich repeat Receptors (NLRs) are key components of plant immune systems. How CC (coiled-Coil)-type NLRs (CNLs) trigger defence signaling is poorly understood. To investigate the role(s) of their CC domains in defense induction we performed extensive genome-wide functional and in silico analyses of nearly all CNLs in Arabidopsis thaliana (At-Col-0). Correlating CC sequence variation with their ability to homo-/heterodimerize and to induce cell death and/or disease resistance in At-Col-0, lettuce and N. benthamiana revealed distinct regions in the $\mathrm{CC}$ required for their function. Reverse genetics in lettuce identified a canonical CNL receptor required for signaling triggered by an At-Col-0 CC. This implies that upon pathogen perception CNLs use their CC domains to transactivate other CNL receptors to form homo and heteromeric assemblies. Surprisingly, a highly variable part of the CC domain is required for this transactivation and partner selection. Together these data suggest that, in contrast to metazoan NLRs, the N-terminal CC domain, rather than the centrally located nucleotide-binding (NB) domain, can mediate initial partner selection. The observed physical and functional associations provide NLR receptors with the ability to form interconnected signaling networks that are expected to have increased resilience to perturbation by pathogens.

Recurrent hybridization introduces high genetic variability in crop pathogen

E. H. STU.K.ENBROCK, Environmental Genomics, Botanical Institute, University of Kiel, Kiel, Germany

Genome analyses have revealed a profound role of hybridization in the evolution of many eU.K.aryote lineages, including fungi. We analyzed signatures of introgression along the genome of a fungal wheat pathogen Zymoseptoria tritici. We applied a comparative population genomics approach, including genome data from five Zymoseptoria species, to characterize the distribution and composition of introgressed regions representing segments with an exceptional haplotype pattern. These regions comprise five percent of the total genome and overlap with $>1000$ predicted genes. We performed window-based phylogenetic analyses along the genome to distinguish regions with monophyletic and nonmonophyletic origin and show that a majority of non-monophyletic windows overlap with the highly variable regions suggesting that these originate from interspecific hybridization events. We verified that incongruent gene genealogies do not result from incomplete lineage sorting (ILS) by comparing the observed and expected length distribution of haplotype blocks resulting from ILS. Protein-coding genes are not enriched in these regions, but we identify 18 encoding putative virulence determinants. We find an enrichment of transposable elements (TEs) in these 
regions implying that hybridization may contribute to horizontal spread of TEs. We detected a similar pattern in the closely related species $Z$. ardabiliae, suggesting that hybridization is widespread among these closely related grass pathogens.

Identification and evolution of transcription factors that govern the biology of oomycete lifestyles S. DE VRIES (1), B. A. Curtis (1), S. L. Harikrishnan (2), L. Sterck (2), Y. Van de Peer (3,4), C. M. Gachon (5), S. A. Rensing (6), C. H. Slamovits (1), (1) Dalhousie University, Halifax, NS, Canada; (2) VIB Center for Plant Systems Biology, Ghent, Belgium; (3) VIB - UGent Center for Plant Systems Biology, Belgium; (4) Department of Plant Biotechnology and Bioinformatics, Ghent University, Belgium; (5) Scottish Association for Marine Science, Oban, U.K.; (6) Philipps University of Marburg, Marburg, Germany

Oomycete plant pathogens can be biotrophs, necrotrophs, or hemibiotrophs. Keeping a host alive (biotrophy) requires a fundamentally different biology than degrading and ultimately killing hosts (necrotrophy). Consequently, oomycetes invoke lifestyle-dependent transcriptional responses upon infection. Yet, despite the biologically distinct expression profiles used, the different lifestyles have evolved multiple times in oomycetes. One explanation might be that recurring evolution of gene regulatory networks (GRNs) drives the evolution of lifestyles. GRNs hinge upon the activity of transcription factors (TFs). To gain insights into the evolution of lifestyle-dependent GRNs, we explored the evolution of oomycete TFs. We screened the TF repertoire of 26 oomycetes and eight other stramenopiles, detecting on average 228 TFs per species. Oomycetes are enriched in $11 \mathrm{TF}$ families, including for example the MADS family associated with sporangia maturation. Of the 11 families, $\mathrm{C} 2 \mathrm{H} 2 \mathrm{and} \mathrm{Myb}-$ related TFs were enriched in hemibiotrophic and necrotrophic pathogens compared to biotrophic ones. Using phylogenetic-based expression analyses, we identified two clades of Myb-related TFs with lifestyle-specific expression: orthologous TFs of hemibiotrophic and necrotrophic plant pathogens have a higher expression than those of animal pathogens and biotrophic plant pathogens. Currently we determine the targets and the evolution of targeting of these TFs across oomycetes.

Dissection of the Tox3-PR1 protein interaction in the Parastagonospora nodorum-wheat interaction

Y. C. SUNG (1), M. A. Outram (2), S. J. Williams (3), P. S. Solomon (4), (1) Research School of Biology, The Australia National University, Canberra, Australia; (2) University of Queensland, Brisbane, Australia; (3) Australian National University, Canberra, ACT, Australia; (4) The Australian National University, Canberra, Australia

The Tox3 effector protein from the pathogen Parastagonospora nodorum elicits a strong necrotic reaction in Snn 3 wheat lines and also interacts with the wheat defence protein PR1. To dissect the biological role of the PR1 protein interaction, we heterologously expressed multiple PR1s from wheat and determined their effect on $P$. nodorum growth in planta. Nearly all PR1 proteins suppressed the growth of the pathogen implying a defence role during disease. Subsequent growth assays confirmed that the recombinant proteins were not anti-microbial but did induce strong defence gene expression in wheat. We demonstrate that this induction of defence signaling is due to the release of the CAPE1 peptide embedded within the C-terminus of PR1 and that defence gene activation is compromised by the interaction of Tox3 and PR1. To dissect this protein interaction, high-resolution crystal structures were generated for Tox3 and several PR1 proteins. We identified a Proline at position 173 on Tox3 was required for interaction and also the repression of PR1-mediated defence signaling. Importantly, the P173 Tox3 mutant protein was not compromised in its ability to induce Snn3-meditaed necrosis demonstrating that the Tox 3 effector is independently able to induce necrosis and also repress host defence. This study has identified the key role of PR1-mediated defence signaling through CAPE1 release and exposed the dual functionality of the Tox 3 effector protein during disease.

Extracellular vesicles as key mediators of plant-microbe interactions

R. W. INNES (1), B. D. Rutter (1), P. Baldrich (2), H. Zand Karimi (1), R. Podicheti (1), B. C. Meyers (2), (1) Indiana University, Bloomington, IN, U.S.A.; (2) Donald Danforth Plant Science Center, Saint Louis, MO, U.S.A.

Exosomes are extracellular vesicles (EVs) that play a central role in intercellular signaling in mammals by transporting proteins and small RNAs. Plants are also known to produce EVs, particularly in response to pathogen infection. Our laboratory has developed methods for purifying EVs, which has enabled us to characterize their contents. These analyses have revealed that plant EVs are highly enriched in proteins involved in biotic and abiotic stress responses and carry miRNAs and siRNAs. They are also highly enriched in single stranded RNAs of 10-15 nucleotides in length, which we have named 'Tiny RNAs' (tyRNAs). These tyRNAs are derived from diverse sources and appear to be degradation products of longer RNA molecules. Recent work has established that EVs are rapidly taken up by filamentous pathogens from host plants and in vitro, and we are currently investigating the mechanism of uptake and the impacts of EVs on fungal physiology. These findings suggest that EVs represent an important component of the plant immune system.

Targeting post-translational modifications to open the black box of pattern recognition receptor signaling

P. Derbyshire (1), L. Grubb (2), S. Jiang (1), Y. Kadota (1,3), J. Sklenar (1), J. Monaghan (1,4), C. Zipfel (1,5), F. L. H. MENKE (1), (1) The Sainsbury Laboratory, University of East Anglia, Norwich Research Park, Norwich, U.K.; (2) Queens Univeristy, Kingston, Canada; (3) RIKEN Center for Sustainable Resource Science, Plant Immunity Research Group, Yokohama, Japan; (4) Queen's University, Kingston, ON, Canada; (5) Inst. of Plant and Microbial Biology, University of Zürich, Zurich, Switzerland

Pattern recognition receptors (PRRs) play a key role in the first line of defense in plant innate immunity. PRR binding of their cognate ligands, so called pathogen-associated molecular patterns (PAMPs), triggers a signaling network that ultimately results in an immune response. Despite recent advances, it has still remained largely an open question how activated PRRs are connected to downstream defense activation. However, it is evident that changes in phosphorylation play a major role and changes in ubiquitination play an emergent role. We have used quantitative phosphoproteomics approaches to identify a large array of verified and potential components involved in PRR signaling. Some of the earliest changes in phosphorylation, immediately downstream of activated PRRs, were identified on receptor-like cytosolic kinase, mitogen-activated protein kinase kinase kinases and calcium dependent protein kinases. Our initial analysis using a ubiquitin-remnant enrichment approach suggests that PRRs as well as immediate downstream signaling components also become rapidly ubiquitinated. To unravel the interplay between these two 
post-translational modifications in PRR signaling, we are using targeted proteomics. By measuring PAMP-triggered temporal changes in both phosphorylation and ubiquitination on components of the PRR signaling network we hope to shed light on the black box in PRR signaling.

Using Virulence Mutants to Identify Avr Genes in the wheat stem rust fungus, Puccinia graminis f. sp. tritici. N. Upadhyaya (1), D. Ortiz (1), F. Li (2), J. Sperschneider (1), X. Zhang (3), M. Luo (1), H. Nguyen-Phuc (2), C. D. Hirsch (2), B. Steffenson (4), K. Silverstein (2), R. Park (5), K. KanyU.K.a (6), J. Rathjen (7), D. A. Jones (3), J. G. Ellis (1), M. Figueroa (2), P. N. DODDS (1), (1) CSIRO Agriculture and Food, Canberra, ACT, Australia; (2) University of Minnesota, St. Paul, MN, U.S.A.; (3) The Australian National University, Canberra, ACT, Australia; (4) University of Minnesota, Saint Paul, MN, U.S.A.; (5) University of Sydney, Cobbitty, NSW, Australia; (6) Department of Biointeractions and Crop Protection, Rothamsted Research, Harpenden, U.K.; (7) Research School of Biology, The Australian National University, Canberra, ACT, Australia

Rust fungi are biotrophic pathogens that depend on living host cells for their growth and form specialised haustoria structures during infection that serve as nutrient uptake sites as well as delivering effector proteins to the host cell. We have been investigating the molecular basis of rust infection and immunity using Melampsora lini (flax rust) as model system and extending this work to Puccinia graminis f. sp. tritici (Pgt) (wheat stem rust) which is one of the most destructive pathogens of wheat. We recently generated a chromosome level genome assembly for $P g t$ based on Pacbio sequence and $\mathrm{HiC}$ scaffolding, which has enabled us to characterise a number of spontaneous virulence mutants. Most result from complex structural rearrangements in the genome, including translocation events between chromosomes and even exchange of chromosomes between the haploid nuclei of this dikaryotic fungus. This has led to identification of several Avr genes from Pgt, as well as development of new tools for testing effector function in wheat including viral overexpression and wheat protoplast transient expression assays. Avr genes in both $M$. lini and Pgt encode secreted proteins that are co-ordinately expressed during infection and have roles in suppressing host defense responses. Using transgenic flax rust we have visualised delivery of AvrM:YFP fusion proteins from haustoria to infected host cells.

Plant immunity and cell death in fungal root symbiosis

A. ZUCCARO, D. Sarkar, H. Rövenich, S. Nizam, G. Langen, University of Cologne, Germany

Plants associate with a wide range of beneficial fungi in their roots which facilitate plant mineral nutrient uptake in exchange for carbohydrates and other organic metabolites. These associations play a key role in shaping terrestrial ecosystems and are widely believed to have promoted the evolution of land plants. To establish compatibility with their host, root-associated fungi have evolved diverse colonization strategies with distinct morphological, functional and genomic specializations as well as different degrees of interdependence. They include obligate biotrophic arbuscular mycorrhizal (AM), facultative biotrophic ectomycorrhizal (ECM) and root endophytic associations, which due to their inconspicuous nature have been often overlooked. Recent research into the biology and genomics of root associations revealed fascinating insight into the phenotypic and trophic plasticity of these fungi and underlined genomic traits associated with biotrophy and saprotrophy. In this talk we will consider the commonalities and differences of AM and ECM associations and contrast them with root endophytes. Additionally, we will present data on the mechanisms driving multipartite interactions and the different colonization strategies of pathogens and mutualistic root associated fungi using a gnotobiotic natural soil-based split-root system for phenotypic and transcriptional analyses.

RPW8/HR Repeats Predict NLR-dependent Hybrid Performance C. A. Barragan (1), D. Weigel (1), E. CHAE (2), (1) Max Planck Institute for Developmental Biology, Tuebingen, Germany; (2) National University of Singapore, Singapore

In many plant species, conflicts between divergent elements of the immune system can cause hybrids to express autoimmunity, a generally deleterious syndrome known as hybrid necrosis. Here we report multiple hybrid necrosis cases in Arabidopsis thaliana that are caused by allelespecific interactions between different variants at two unlinked resistance $(\mathrm{R})$ gene clusters. One is the RESISTANCE TO PERONOSPORA $P A R A S I T I C A 7$ (RPP7) cluster, which encodes an intracellular nucleotide binding site-leucine rich repeat (NLR) immune receptors that confer strain-specific resistance to oomycetes. The other is the RESISTANCE TO POWDERY MILDEW 8 (RPW8)/HOMOLOG OF RPW8 (HR) locus, which encodes atypical resistance proteins that can confer broad-spectrum resistance to filamentous pathogens. There is extensive structural variation in the $R P W 8 / H R$ cluster, both at the level of gene copy number and at the level of C-terminal protein repeats of unknown function. We demonstrate that the number of RPW8/HR repeats correlate, albeit in a complex manner, with the severity of hybrid necrosis when these alleles are combined with specific $R P P 7$ variants. This observation suggests that gross structural differences, rather than individual amino acid polymorphisms, guide the genetic interaction between $R P W 8 / H R$ and $R P P 7$ alleles. We discuss these findings in light of the similarity of RPW8/HR proteins with pore-forming toxins, MLKL and HET-S, from mammals and fungi.

The role of chloroplasts in disease and defence.

T. Gaikwad (1), S. Breen (1), S. Kulasekaran (1), D. Arnaud (2), R. F. Hussain (1), N. Smirnoff (2), E. Breeze (1), J. R. Alfano (3), G. Littlejohn (4), M. R. GRANT (5), (1) The University of Warwick, Coventry, U.K.; (2) The University of Exeter, Exeter, U.K.; (3) University of Nebraska, Lincoln, NE, U.S.A.; (4) University of Plymouth, Plymouth, U.K.; (5) University of Warwick, Warwick, U.K.

Chloroplasts are emerging as key integrators of biotic stress responses. In addition to their photosynthetic capacity, chloroplasts are involved in the synthesis of phytohormone precursors and generate a range of reactive oxygen species, both key signaling components that determine plantpathogen outcomes. Our research explores the role of the chloroplast in disease and defence, focussing on early events following microbe associated molecular pattern recognition and how chloroplasts are targeted by effectors to suppress innate immunity using the Arabidopsis Pseudomonas syringae pathosystem. We are particularly interested in how pathogen effectors directly or indirectly target chloroplasts to suppress reactive species and how chloroplasts may interact with other organelles to elaborate a defence response. Furthermore, consistent with an emerging role for the chloroplast in effector triggered immunity, we will present recent findings that implicate the chloroplast in the generation of a long-distance signal(s) that drives systemic acquired resistance. 
Proline-rich extensin-like receptor kinases mediate damage-triggered immune responses to nematode infections J. L. LOZANO TORRES, J. J. Willig, S. Warmerdam, C. C. Van Schaik, J. Bakker, A. Goverse, G. Smant, Laboratory of Nematology, Wageningen University and Research, Wageningen, Netherlands

Plant-parasitic nematodes constitute a major threat to global food production. Despite causing considerable damage to host tissue during parasitism, little is known on the role of plant basal immunity in resistance to nematodes. We have recently discovered that suppression of basal immunity to nematodes involves a specific class of surface-localized receptors i.e. proline-rich extensin-like receptor kinases (PERKs). However, the role of PERKs as mediators of damage-triggered basal immunity is not well understood. PERKs are differentially regulated upon infections with cyst and root-knot nematodes. Arabidopsis perk-mutants show increased susceptibility to both cyst and root-knot nematodes. By contrast overexpression of AtPERK-A results in enhanced resistance to cyst nematodes. Furthermore, Arabidopsis lines heterologously overexpressing AtPERK-A homologs also show enhanced resistance to cyst nematode infections. AtPERK-A is strongly induced in nematode feeding sites and neighbouring cells. The basal immune response mediated by PERK-A during nematode infection involves binding of the extracellular domain to polygalacturonic acid and the downstream regulation of innate immune suppressors. We, therefore, conclude that PERKs function as damagetriggered immune receptors during nematode infections and as such can be exploited as a novel source of nematode resistance.

How do aphids promote plant susceptibility?

J. I. B. BOS, University of Dundee, Dundee, U.K.

Aphids are devastating insect pests of crops globally and, therefore, pose a major threat to food security. These insects deliver effectors inside host plants during probing and feeding to suppress the plant immune system and enhance susceptibility. We used a combination of "omics" approaches to identify and compare effector repertoires from economically important aphid species. We generated new insight into how aphid genomes, including effector repertoires, have evolved and revealed evidence for a shared transcriptional control mechanism, which underlies effector expression, and ultimately plant parasitism. Functional characterisation of select aphid effectors revealed that these proteins associate with host plant proteins, and that these associations are linked to effector virulence activity, pointing to important parallels between plant-insect and plant-microbe interactions. Our work shows that aphid effectors associate with host proteins of diverse functions in cellular trafficking, redox regulation and post-translational modification. Further characterisation of these associations will reveal not only how effectors contribute to enhancing host susceptibility, but also will provide novel insight into plant biological processes targeted during infestation. This in turn will enable the development of novel protection strategies against aphids that are durable and sustainable.

Structural analysis for a 1-piperidine-2-carboxylic acid reductase reveals functional conservation of a pipecolic acid pathway in systemic acquired resistance

P. DING (1), M. Torrens-Spence (2), J. D. Jones (1), J. K. Weng (2), (1) The Sainsbury Laboratory, University of East Anglia, Norwich Research Park, Norwich, U.K.; (2) Whitehead Institute for Biomedical Research, MIT, Cambridge, MA, U.S.A.

Systemic acquired resistance (SAR) is an induced immune response instigated across the entire plant following a primary infection. In Arabidopsis, this process requires pipecolic acid (Pip) accumulation, which is synthesized from the precursor 1-piperidine-2-carboxylic acid (P2C) by a reductase PCAR, SAR Deficient 4 (SARD4). Here, we report the crystal structure of Medicago truncatula PCAR to give the first insight into the structural basis of PCAR function in plants. MtPCAR contains two domains with distinct functions. The N-terminal dimerization domain is highly conserved in all the mu-crystallin-like protein families and exists in mammals and many prokaryotes. The other domain contains a evolutionarily ancient Rossmann fold that is widely distributed in NAD/NADP-binding enzymes. Unlike other previously solved mu-cyrstallinlike structures, two active site loops adopt unique conformations in MtPCAR structure and likely impact P2C-and NADP-binding and catalysis. We predict that conserved amino acids in these regions are important for the functionalization of MtPCAR from an ancesterial mu-crystallin and will be the target of mutational analysis. Substitutions at these amino acids have also been implicated in the lysine cyclodeaminase, a homolog from Streptomyces pristinaespiralis and likely impact substrate recognition and catalytic activity within the broader mu-crystallin family. Our results further reveal that PCARs in both dicots and monocots are functionally conserved.

Parasites within parasites: How transposable elements drive the evolution of plant pathogenic fungi D. CROLL, University of Neuchâtel, Neuchâtel, Switzerland

Transposable elements are core constituents of eU.K.aryotic genomes including the genomes of most plant pathogens. Transposable elements are intricately associated with pathogenicity, because transposable elements and pathogenicity genes often share similar chromosomal locations. Transposable elements also generated key adaptive genetic variation in pathogen populations. Hence, transposable elements and their plant pathogen host genomes are on complex evolutionary trajectories. To resolve these trajectories, we use large population genomic datasets of the major wheat pathogen Zymoseptoria tritici. First, we analyze co-regulation of transposable elements and pathogenicity genes over the course of a plant infection. We find that stress imposed by host immunity induces de-repression of transposable elements. However, the synchronicity with pathogenicity genes depends on the nature of the transposable element and the genetic background of the pathogen genome. Then, we analyze complete genomes of isolates spanning the worldwide distribution and show that transposable element activity explains a significant fraction of all intra-specific structural variation. We find that the demographic history of the pathogen likely had an impact on transposable element control contributing to extant population differentiation. In conjunction, our results show that transposable elements impose strong trade-offs on plant pathogen evolution by reshaping the genome structure and promoting adaptation.

Colletotrichum metabolite higginsianin B suppresses plant jasmonate signaling by blocking proteasome-mediated degradation of JAZ proteins

J. F. Dallery (1), V. Halder (2), G. Le Goff (1), D. D. Gianniou (3), I. P. Trougakos (3), J. Ouazzani (1), D. Gasperini (4), R. J. O’CONNELL (5), (1) CNRS Institut de Chimie des Substances Naturelles, Gif-sur-Yvette, France; (2) Max Planck Institute for Plant Breeding Research, Cologne, 
Germany; (3) National and Kapodistrian University of Athens, Greece; (4) Leibniz Institute of Plant Biochemistry, Halle, Germany; (5) INRA BIOGER, Thiverval-Grignon, France

The hemibiotrophic fungus Colletotrichum higginsianum causes anthracnose disease on Brassicaceae. Its genome contains 77 secondary metabolite biosynthetic gene clusters (BGCs) but the nature and functions of their chemical products are largely unknown. To produce sufficient metabolites in axenic cultures for use in functional studies, we deleted the chromatin regulator CclA that negatively regulates BGCs through H3K 4 trimethylation. Ccla mutants overproduced 3 families of terpenoids, allowing the isolation of 11 molecules, of which 5 were new. The pure compounds were then tested in combination with MeJA, an elicitor of jasmonate responses, for their ability to alter defense gene induction in Arabidopsis. One molecule, higginsianin B (hiB), inhibited MeJA-triggered expression of the defense reporter VSP1p:GUS, suggesting hiB may block bioactive JA-Ile synthesis or signaling in planta. By using the JA-Ile sensor Jas9-Venus, we found hiB inhibits JA-Ile signaling by preventing the degradation of JAZ proteins, the repressors of JA responses. HiB likely blocks $26 \mathrm{~S}$ proteasome-dependent JAZ degradation, as it inhibited chymotrypsin- and caspase-like protease activities. The dose-dependent inhibition of target degradation by hiB also extended to auxin signaling, as hiB treatment reduced IAA-dependent expression of DR5p:GUS. Overall, our data show specific fungal secondary metabolites can act similar to protein effectors to subvert plant immune and developmental responses.

Cooperation between hyphal cells underpins plant colonization by the fungal pathogen Sclerotinia sclerotiorum S. RAFFAELE (1), A. Barbacci (2), M. Mbengue (3), R. Peyraud (2), (1) Laboratory of Plant-Microbe Interactions, Toulouse, France; (2) INRA, France; (3) LRSV Université de Toulouse, France

Cooperation between specialized units supports complex biological functions, from the colonization of unfavorable environments to the formation of organs and sociality. Filamentous fungi are composed of strings of cells growing from the tip (hyphae) exposed to heterogeneous environments. Successful fungal pathogens are able to fend off host defenses while maintaining growth and foraging for nutrients. By examining the global regulation of genes in different areas of colonies of the fungal plant pathogen Sclerotinia sclerotiorum, we found evidence for cooperation between cells along hyphae. Gene expression patterns diverged markedly in cells at the center and apex of hyphae during the colonization of Arabidopsis thaliana plants compared to in vitro growth. We reconstructed a genome-scale metabolic model for S. sclerotiorum and used flux balance analysis to demonstrate metabolic heterogeneity supporting division of labor between hyphal cells. Using a multi-cell model of fungal hyphae, we show that the gain in invasive growth conferred by cooperation increases with host resistance. Ablation assays and in planta growth measurements provided experimental validation for major predictions from our models, identifying cooperation in fungal hyphae as a mechanism emerging at the multicellular level to support host colonization and virulence. Progress in the analysis of mechanisms controlling division of labor in S. sclerotiorum will be presented.

Contrasting colonization and host range of Sphingomonas and Pseudomonas in wild Arabidopsis thaliana and neighboring plants D. S. LUNDBERG (1), R. de Pedro Jové (2), P. Pramoj Na Ayutthaya (3), T. Karasov (1), O. Shalev Skriptchak (1), K. Poersch (1), D. Weigel (1), (1) Max Planck Institute for Developmental Biology, Tuebingen, Germany; (2) Centre for Research in Agricultural Genomics (CRAG), Barcelona, Spain; (3) Interfaculty Institute of Biochemistry, Tuebingen, Germany

The bacterial genera Pseudomonas and Sphingomonas dominate the phyllosphere of wild Arabidopsis thaliana. Many Pseudomonas isolates from A. thaliana are pathogenic, reaching high titers in wild plants, while Sphingomonas do not reach such loads but are more consistent in abundance. Prior work showed that some can protect against pathogens. Many members of both genera seem to be commensals. We compare the genomes of hundreds of endophytic Sphingomonas isolates collected from a wild population of $A$. thaliana to investigate intra- and inter-plant strain diversity, and contrast this with Pseudomonas diversity in the same plants, which previous research has shown are colonized primarily by a putatively pathogenic lineage. To help understand the persistence of strain-level diversity, we surveyed the local plant host range of these strains. We used a new approach, culturing both genera in bulk from $A$. thaliana and other plant families, in spring and also in summer before the germination of the next cohort of $A$. thaliana. We sequenced the bulk cultures as metagenomes and mapped reads to local reference genomes. These data, together with $16 \mathrm{~S}$ rDNA amplicon data of the plants and genomes of bacteria isolated from the other plant species, reveal that common bacterial strains on A. thaliana thrive on other local hosts. This suggests that other hosts may be equally or more important for the evolution of these bacteria, despite the great success of these strains on A. thaliana.

Molecular determinants of host specialization in grass powdery mildews

B. KELLER, S. Bourras, L. Kunz, M. C. Müller, C. R. Praz, B. Manser, University of Zurich, Zurich, Switzerland

The grass powdery mildew pathogen occurs in different forms that have very specific host ranges. Based on phylogenetic and genomic analysis, host jumps and host expansions have been described in grass powdery mildew. We want to understand the molecular basis of host specialization and host expansion in the different formae speciales of mildew. We have recently identified at the molecular level several avirulence genes recognized by the allelic series of $P m 3$ resistance genes in wheat. We have characterized five effector proteins from three mildew forms specialized on wheat, rye and the grass Dactylis glomerata that are specifically recognized by the PM3B, PM3C and PM3D immune receptors in wheat. Furthermore, non-host infection assays demonstrate that $P m 3 b, P m 3 c$, and $P m 3 d$ are restricting growth of rye mildew on wheat. Together, these observations suggest an involvement of AVRPM3B/C/D in host specificity. These studies on the role of specific avirulence genes in host specialization are complemented by an unbiased genetic approach to study the basis of a recent host expansion of powdery mildew to the novel crop Triticale which is an amphiploid of rye and wheat. There, we found that pathogen adaptation resulted from hybrid formation of rye and wheat powdery mildews, mirroring host evolution of Triticale. We are currently identifying the molecular factors underlying this host adaption using a high-resolution segregating mildew population.

Dual regulation of reactive oxygen species through direct ubiquitination and phosphorylation of the plant immune regulator RBOHD D. LEE, University of California, Davis, CA, U.S.A. 
Generation of reactive oxygen species (ROS) is a critical component for innate immune responses against pathogen infection. The Arabidopsis NADPH oxidase RBOHD is a key ROS producing enzyme in response to pathogen perception. Excessive ROS production of can negatively impact host development and viability, thus ROS production must be tightly regulated at a resting state. Here we show that RBOHD's stability is reduced by the receptor-like protein kinase PBL13. PBL13 phosphorylates the C-terminus of RBOHD at six conserved residues in vitro. Genetic and biochemical analyses revealed that two PBL13 phosphorylation sites are required for RBOHD activity and stability. Interestingly, Mimicking phosphorylation of RBOHD by PBL13 enhances ubiquitination of RBOHD in vivo. Using protein array technology, we identified a novel E3 ubiquitin ligase PIRE (PBL13 interacting RING domain E3 ligase) that interacts with both PBL13 and RBOHD. PIRE specifically ubiquitinates RBOHD but not PBL13. PIRE and PBL13 mutants display enhanced RBOHD abundance, elevated ROS production and enhanced resistance to bacterial infection. Taken together, our study provides valuable insights on undefined post-translational mechanisms involved in ROS production through regulating the stability of a conserved NADPH oxidase during host-pathogen interaction.

Genetic engineering of the Brassicaceae smut fungus Thecaphora thlaspeos and analysis of its RNAi system K. BÖSCH (1), M. Feldbrügge (2), V. Goehre (1), (1) Heinrich-Heine University, Duesseldorf, Germany; (2) Heinrich-Heine-Universität, Düsseldorf, Germany

Smut fungi infect economically important crop plants, e.g. maize or potato. To protect these, we need to understand the infection process on a molecular level. When compared to crop plants, Brassicaceae offer simpler genetics enabling easier analysis of plant responses. Therefore, we want to characterize the molecular interaction between the newly described Brassicaceae smut fungus Thecaphora thlaspeos in its host plants Arabidopsis thaliana and Arabis alpina. To gain access to the fungal side, a transformation system of haploid filamentous cultures is established. Constructs can be integrated randomly into the genome giving rise to different marker strains. Currently, we are establishing knock-out and knock-down strategies. As a proof of principle, we are targeting a putative mating pheromone gene. Furthermore, the characterization of the RNAi machinery is of special interest, because it is absent in the model smut fungus Ustilago maydis. To investigate its role in smut fungi, we will knock out the only copy of dicer in $T$. thlaspeos. The functionality and usage of the RNAi system in $T$. thlaspeos will be further assessed via sRNA sequencing and silencing of $g f p$ constructs by genomically encoded or exogenously supplied RNAs. This will reveal its contribution to fungal and infection biology.

A Ralstonia solanacearum TALE-like protein co-opts an ancient translational control element to boost host polyamine levels T. LAHAYE, ZMBP, Tübingen, Germany

Transcription activator-like effectors (TALEs) of the bacterial phytopathogen Xanthomonas bind to effector binding elements (EBEs) present in host promoters and activate downstream susceptibility $(S)$ genes to promote disease. We present here the identification of the first plant $S$ gene being activated by a TALE-like effector from the broad host range pathogen Ralstonia solanacearum (de Lange, 2013; Schandry, 2016). Using a synergistic combination of RNA-Seq, computational prediction, promoter-reporter assays, and CRISPR-mutagenesis, we found that the $R$. solanacearum TALE-like protein Brg11 exclusively targets arginine decarboxylase (ADC) genes in tomato. The $E B E$ targeted by Brg 11 is part of the $A D C$-box, a $\sim 50$ bp motif upstream of $A D C$ genes that is conserved across land plant species. Unlike known $E B E s$, the $A D C$-boxis transcribed and functions as a cis-element that attenuates translation. Brg11 induces truncated $A D C$ transcripts lacking the $A D C$-box, bypassing translational control. Since ADCs are rate limiting anabolic enzymes in polyamine (PA) biosynthesis, their elevated translation boosts host PA levels. We show that plant ADCs are components of a plant immune pathway, that likely inhibits growth of microbes that are detrimental to $R$. solanacearum. Our data, in combination with available literatures also suggest that PA levels represent a central hub that is targeted by plantassociated microbes to manipulate plant immune reactions for their own altruistic interest.

Network biology and predictive modeling to link genome to phenome

S. MU.K.HTAR, University of Alabama at Birmingham, Birmingham, AL, U.S.A.

Networks consist of systems' components, referred to as 'nodes', and interactions between them, termed 'edges'. The understanding of how networks function in the schema of an entire system is fueling the development of network-based approaches. Such a framework is pertinent to evaluation of diverse biological networks co-expression, TF-target and protein-protein interactions. Network analysis has been a recent focus in biological sciences due to its ability to synthesize global visualizations of cellular processes and predict functions based on inferences from network properties. Understanding these topological features and deciphering the network architecture can provide insights into the identification of new community structures, unknown signaling pathways, and novel relationships between genes and their products. The work in my laboratory is focused on how macromolecular networks control biological processes and how environmental perturbations in such networks can explain diverse phenotypes. We integrate various layers of -omics to understand genome to phenome relationships.

AVR2-mediated resistance to Phytophthora infestans by unrelated $R$ genes from wild Solanum species

C. AGUiLERA GALVEZ (1), H. Wang (2), E. M. Gilroy (2), Z. Chu (3), D. Wouters (1), J. H. Vossen (1), R. G. F. Visser (1), J. D. Jones (4), P. R. J. Birch (5), V. G. A. A. Vleeshouwers (1), (1) Plant Breeding, Wageningen University and Research, Wageningen, Netherlands; (2) The James Hutton Institute, Dundee, U.K.; (3) The Sainsbury Laboratory, Norwich Research Park, Norwich, U.K.; (4) The Sainsbury Laboratory, University of East Anglia, Norwich Research Park, Norwich, U.K.; (5) Cell and Molecular Sciences, James Hutton Institute, Dundee, U.K.

Late blight caused by the oomycete Phytophthora infestans remains the most devastating disease for potato. Wild Solanum species provide a valuable resource of resistance $(R)$ genes. Yet, for wise $R$ gene stewardship and deployment, molecular knowledge of the corresponding avirulence $(A v r)$ gene is essential. AVR2 is an RXLR effector of $P$. infestans with an important role in late blight virulence. AVR2 is recognized by the R2 family from wild Mexican Solanum species and by the dissimilar Rpi-mcql family from Peruvian S. mochiquense. Thus, two sequenceunrelated R proteins recognize the same effector. Interestingly, the recognition of AVR2 by Rpi-meq1 and R2 displays an overlapping, but not identical specificity. Disease tests with a wide panel of $P$. infestans isolates on transgenic $R 2$ and Rpi-mcql potatoes show different patterns of disease resistance (Aguilera-Galvez et al, 2018. Studies in Mycology). Studies of the AVR2 family revealed that this is a highly diverse effector 
family that is differentially expressed in $P$. infestans isolates. We found new AVR2 alleles and we hypothesize that the AVR2 family is coevolving with two different $R$ gene families. These studies contribute to understanding the R-AVR interaction, which is crucial to improve $R$ gene-based breeding and deployment against late blight in potato.

Evolution of the LATD/NIP meristem function in roots and nodules.

J. M. HARRIS, G. Sassi, B. M. Tinaz, S. T. Connolly, Z. M. Portlas, University of Vermont, Burlington, VT, U.S.A.

The LATD/NIP gene of Medicago truncatula is required for root and nodule meristem function; this common requirement indicates a shared genetic component and supports the idea that lateral roots and nodules are homologous organs. Thus, the evolutionary history of the LATD/NIP gene may provide insight into the evolution of nodule development. LATD/NIP encodes a nitrate transporter of subfamily 1 of the large NPF family. Interestingly, although other NPF genes control root growth, LATD/NIP/MtNPF1.7 is the only one essential for meristem function. At what point did $L A T D / N I P$ acquire its root and nodule meristem function? Was this function acquired all at once, or were root and nodule meristem functions acquired sequentially? Phylogenetic analysis of NPF1 reveals 5 clades, caused by sequential gene duplication. LATD/NIP originated at the base of the eudicots, well before the nitrogen-fixing clade, and is retained specifically in taxa that form mycorrhizal and nodule symbioses, suggesting a role for NPF1 genes in root symbioses. To investigate the origins of $L A T D / N I P$ meristem function, we are testing whether $L A T D / N I P$ orthologs from other taxa are able to functionally complement the Mtlatd mutant, and also characterizing the phenotype of Lotus japonicus latd/nip mutants. We find that the LATD/NIP root meristem function was present prior to the origin of the Nitrogen-fixing clade, and thus was acquired before the evolution of nitrogen-fixing root nodules.

Oomycetes: A new episode of cross kingdom RNA interference

F. DUNKER, A. Weiberg, Ludwig-Maximilians University (LMU), Munich, Germany

Oomycetes contain devastating plant pathogens. Mobile small RNAs (sRNAs) can trigger RNA silencing across kingdoms in plant-fungal and plant-insect interactions. Here, we investigated cross kingdom RNA interference (RNAi) in the interaction of the downy mildew pathogen Hyaloperonospora arabidopsidis (Hpa) and its host Arabidopsis thaliana (At). We infected At RNAi pathway mutants and found that exclusively atagol mutants revealed a reduced infection phenotype; trailing necrosis around hyphae and lower pathogen spread. As no other plant microRNA or small-interfering RNA biogenesis mutant showed reduced susceptibility, we profiled sRNAs binding to AtAGO1 during Hpa infection by AGO1 immunopurification coupled to sRNAseq. We found that $>100$ HpasRNAs bound the plant AGO1 and target prediction of HpasRNAs included several host genes putatively involved in pathogen defense. We elucidated that the At With No Lysine(K) (WNK) Kinase 2 and the Aspartyl Protease AtAED3 were indeed silenced, as shown by gene expression analysis and 5' RACE-PCR. Further, atwnk2 and ataed 3 loss-offunction mutants promoted higher $\mathrm{Hpa}$ sporangiophore and spore production, indicating a gene function in pathogen defense. To clarify the role of HpasRNAs during host colonization, we generated transgenic plants expressing short-tandem target mimics (STTM) sequestering HpasRNAs. These mutant plants showed reduced Hpa disease demonstrating cross kingdom RNAi is a virulence mechanism in a pathogenic oomycete.

FERONIA and the regulation of receptor kinase-mediated signaling during immunity and beyond

C. ZIPFEL, Inst. of Plant and Microbial Biology, University of Zürich, Zurich, Switzerland

Plants genomes encode hundreds of cell surface-localized receptor kinases (RKs) that control almost all aspects of plant life, ranging from reproduction, growth to responses to the external environment. Using RKs that function as immune receptors by perceiving microbial elicitors, we are studying the molecular basis of plant immunity, but also more generally how plant RKs work at the mechanistic level. Using the leucinerich repeat RKs FLS2 and EFR (which perceive bacterial flagellin and EF-Tu, respectively) as model systems, we are investigating how plant RKs function as part of multimeric protein complexes at the plasma membrane - often in complex with other RKs, which act as helper proteins. Our recent work uncovered the importance of these helper RKs and RK-associated proteins in controlling the assembly of functional heteromeric receptor complexes. These observations also raise the inherent question of how these dynamic receptor complexes get formed and organized at the plasma membrane. Notably, we have recently uncovered an important role of the Catharantus roseus RLK1-like (CrRLK1L) FERONIA (FER) as a helper RK controlling immunity. Building on this recent published work, I will present our unpublished work that sheds light on the mechanisms underlying ligand perception by FER, and on the more general role that FER may play in the regulation of multiple signaling pathways beyond immunity and reproduction.

The root endophyte Serendipita vermifera modulates extracellular nucleotide levels to transition from biotrophy to cell death-associated root colonization

H. RÖVENICH, H. Widmer, S. Nizam, S. Wawra, G. Langen, A. Zuccaro, University of Cologne, Germany

Apoplastic nucleotides play an important role in plant-fungus interactions. We have recently shown that the beneficial endophyte Serendipita indica secretes an ecto-5'-nucleotidase ( $\mathrm{SiE} 5^{\prime} \mathrm{NT}$ ) during root colonization. SiE5'NT hydrolyses nucleotides in the apoplast, and Arabidopsis thaliana lines producing extracellular SiE5'NT are significantly better colonized. The overexpression of SiE5'NT coincides with reduced levels of extracellular adenosine $5^{\prime}$-triphosphate (eATP), which accumulates during early colonization stages when $S$. indica maintains a biotrophic lifestyle. Here, we report that the synergistic action of SiE5'NT and a second extracellular fungal enzyme, S. indica nuclease A (SiNucA), activates a metabolic cell death mechanism in A. thaliana. Since extracellular nucleotide and nucleoside pools are affected in the apoplast during root colonization by Sebacinoid fungi, we hypothesise that a link exists between programmed cell death and Sebacinoid-associated metabolic changes in planta.

Molecular Interactions between Fusarium graminearum and Host Plants

W. TANG, D. Zhang, Shanghai Institute of Plant Physiology and Ecology, CAS, Shanghai, China 
The ascomycete fungus Fusarium graminearum not only infects wheat to cause Fusarium head blight and seedling blight, but also infects maize to cause Gibberella stalk rot. In addition, it can also grow in axenic media. To comprehensively understand how $F$. graminearum invades various hosts, various tissues and causes different diseases, we take an approach of cellular tracking and gene profiling of fungal infection process. We tracked $F$. graminearum growth inside host plants during disease development and found fungal growth inside hosts has different paths: intercellularly and intracellularly. Using laser microdissection, we profiled in planta fungal gene expression during wheat seedling coleoptile infection and during maize stalk infection in a stage-specific manner and started to elucidate its molecular strategies in confronting the host environments. In wheat infection, we identified that a nonribosomal peptide, as a product of secondary metabolite biosynthesis cluster of $F$. graminearum, facilitates cell-to-cell invasion in wheat, probably through manipulating plasmodesmal permeability and/or chloroplast activity. In maize stalk disease progression, we showed that $F$. graminearum remodels membrane lipids to overcome the phosphate limitation in the intercellular space of maize stalks.

Kilbournase, a protease-associated domain subtilase secreted by the fungal corn pathogen Stenocarpella maydis T. A. NAUMANN (1), M. J. Naldrett (2), N. P. Price (1), (1) USDA ARS NCAUR, Peoria, IL, U.S.A.; (2) University of Nebraska - Lincoln, Lincoln, NE, U.S.A.

The protease-associated (PA) domain is structurally separate from but encoded within the active site of some large subtilisin proteases. The most well-characterized PA domain subtilase is ScpA, a Streptococcal cell wall protease that truncates the human immunity protein C5a. PA domains are thought to give substrate specificity to subtilisins by capping the active site. Here we demonstrate that a protease secreted by the fungal corn pathogen Stenocarpella maydis, which truncates the corn ChitA protein, is a PA domain subtilase. Purified protease was isolated from $S$. maydis cultures and tryptic peptides were analyzed by LC-MS/MS. Observed peptide ions were mapped to two hypothetical PA domain subtilases encoded by the $S$. maydis genome. Yeast strains were created to express each candidate. One strain produced a protease that truncated ChitA. This protease was purified from yeast and its activity was characterized biochemically. It degraded the amino-terminal domain of corn ChitA, while leaving the catalytic domain intact. It did not modify a homologous chitinase from the dicot plant Arabidopsis thaliana, indicating a high level of substrate specificity. The identified protein, that we name kilbournase, is only the second described subtilase that is an exoprotease. Truncation of corn ChitA by this pathogen PA domain subtilase, which resembles Streptococcal ScpA, suggests ChitA may play a role in innate immunity.

Pathogen effector recognition by NLR immune receptors with integrated decoy domains

T. KROJ (1), Y. Xi (2), D. Ortiz (3), L. Guo (4), J. Liu (4), K. D. Guillen (5), A. Padilla (5), S. Cesari (2), (1) INRA Montpellier, Montpellier, France; (2) BGPI, Univ Montpellier, CIRAD, INRA, Montpellier SupAgro, Montpellier, France; (3) CSIRO Agriculture and Food, Canberra, ACT, Australia; (4) China Agricultural University, Beijing, China; (5) CBS, Univ Montpellier, CNRS, INSERM, Montpellier, France

NLRs are an important class of plant immune receptors that mediate specific recognition of pathogen effectors inside host cells by the formation of protein complexes. They are characterized by a multi-domain architecture composed of a variable $\mathrm{N}$-terminal domain, a central nucleotidebinding (NB-ARC) domain and C-terminal leucine-rich repeats (LRR). Based on our work on the detection of the Magnaporthe oryzae effectors AVR-Pia and AVR1-CO39 by the rice NLR RGA5, we developed the hypothesis that some NLRs recognize effectors through non-canonical integrated domains (IDs) that act as mimics of effector targets. By detailed structure-function analysis, we deciphered the molecular details of the binding of AVR-Pia and AVR1-CO39 to the integrated heavy metal-associated (HMA) decoy domain of RGA5 and demonstrated that the direct is strictly required for effector recognition. However, RGA5-HMA/effector binding is only of moderate affinity and acts in concert with the association of the effectors to other sites in RGA5. This combination of integrated decoy domains with additional independent effector-NLR interactions seems to confer robust effector recognition and resilience to effector mutations.

\section{Syringopeptide Phytotoxin is Involved in the Biofilm Formation of Pseudomonas fuscovaginae, Causing Bacterial Brown Sheath Rot} Disease of Rice

Y. LEE (1), O. Choi (2), B. Kang (3), S. Kim (1), J. Kim (1), D. Son (1), (1) Department of Plant Medicine, Gyeongsang National University, Jinju, Korea, Republic of (South); (2) Institute of Agriculture \& Life Science, Gyeongsang National University, Jinju, Korea, Republic of (South); (3) Division of Applied Life Science (BK21 Plus), Gyeongsang National University, Jinju, Korea, Republic of (South)

Pseudomonas fuscovaginae is known as a bacterial pathogen in rice as well as being pathogenic to other gramineae crops including maize, sorghum, and wheat. The object of this study was to isolate and identify the key pathogenic factors of $P$. fuscovaginae PF3, isolated from sheath rot lesions of rice. Using the transposon (Tn) mutagenesis, Tn-based mutants were generated. To find virulence-deficient mutants, rice plant inoculations were used. Biofilm deficient mutants were also screened. Among 1,200 mutant strains, 10 mutants showing virulence deficiency were isolated. Similarly, biofilm deficient mutants were screened. Among 1,300 mutants, 10mutants exhibiting biofilm deficiency were isolated. After cloning and sequencing Tn flanking region, Tn insertion sites were identified. Interestingly, the Tn flanking region of all mutants was found to be inserted in the region of syringopeptide phytotoxin genes. These results indicated that the key virulence factor of $P$. fuscovaginae is syringopeptide phytotoxin, which plays an important role in biofilm formation. To our knowledge, this is the first report demonstrating syringopeptide phytotoxin is involved in the biofilm formation of $P$. fuscovaginae.

Activation of root legume endosymbioses program

P. Dangeville, M. CHARPENTIER, John Innes Centre, Norwich, U.K.

Nuclear localized calcium oscillations are at the core of the common endosymbiotic pathway leading to root nodule and arbuscular mycorrhizal symbioses. Although these nuclear-localized calcium oscillations are essential for the activation of the endosymbiotic program, the precise mechanism that encodes the calcium signals is still poorly understood. Three components involved in the generation of calcium oscillations have been identified. Those include the potassium permeable channel, so called DMI1, and a SERCA-type calcium ATPase, so called MCA8, and the calcium channel encoded by the cyclic nucleotide gated channels CNGC15s. A mathematical modelling approach revealed that those components 
are essential and sufficient to recapitulate the calcium oscillation. Progress in understanding the mechanism of regulation and activation of these components will be presented.

Monoterpenes act as volatile cues in intra-plant and inter-plant propagation of innate immunity M. Wenig (1), A. Ghirardo (2), J. Sales (3), J. P. Schnitzler (2), C. VLOT (3), (1) Institute of Biochemical Plant Pathology, Helmholtz Zentrum Muenchen, Germany; (2) Helmholtz Zentrum Muenchen, Research Unit Environmental Simulation, Germany; (3) Helmholtz Zentrum Muenchen, Institute of Biochemical Plant Pathology, Germany

Monoterpenes are volatile organic compounds that are essential for intra-plant systemic acquired resistance (SAR), a pathogen-induced defense response in tissues that are systemic to the site of infection. SAR further relies on salicylic acid (SA) and LEGUME LECTIN-LIKE PROTEIN1 (LLP1), which promotes SAR in parallel with SA. Experiments using petiole exudates showed that LLP1 is necessary for the establishment of resistance in systemic, SAR signal-receiving tissues. In addition, LLP1 is necessary for both emission and recognition of monoterpenes, which act as volatile cues and propagate SAR signaling between neighboring plants. Consequently, we observed LLP1-dependent positive feedback regulation of monoterpenes turning signal-receiving plants into new 'senders' in the absence of infection. From these data, we hypothesize that monoterpenes as pathogen-inducible plant volatiles form self-fortifying cues with the potential to generate a wave of innate immune signaling within leaf canopies or dense plant stands. Notably, in addition to LLP1, multiple signaling partners of the SAR signaling network promote feedforward regulation of these volatile defense cues. At the same time, LLP1 interacts with jasmonic acid (JA)-associated biotic and abiotic stress responsiveness, raising the possibility that monoterpene-dependent inter-plant innate immune signaling is subject to SA-JA cross talk. Repercussions on the influence of SA, monoterpenes, LLP1, and JA on SAR will be discussed.

From the Microbiome to the Gene: Mapping the genes in a leaf microbiome responsible for strain-specific pathogenicity T. KARASOV, M. Neumann, A. Duque, H. Ashkenazy, D. Weigel, Max Planck Institute for Developmental Biology, Tuebingen, Germany

Microbiome sequencing provides a snapshot of the composition of a microbial community. Determining which genes and functions enable specific taxa to proliferate within a microbial community remains a central challenge. In this study, we aim to determine the genes that increase the proliferation of specific Pseudomonas in the microbiomes of wild Arabidopsis thaliana populations. We recently found that the most abundant Pseudomonas taxon in A. thaliana in Southwestern Germany was composed of genotypically and phenotypically diverse strains. Closely related strains - indistinguishable via $16 \mathrm{~S}$ amplicon sequencing - show a range of pathogenicity phenotypes. Here we combine microbiome analysis, whole genome sequencing of strains, genome-wide association mapping and saturation screening to identify those genes important for strain success. The frequent gain and loss of these genes underscores the capacity for rapid evolution in Pseudomonas

Identification of Rwt6, a wheat resistance gene corresponding to PWT6 which is distributed in Eleusine and Oryza isolates of Pyricularia oryzae.

S. ASU.K.E (1), Y. Umehara (1), M. Iwakawa (1), Y. Tosa (2), (1) Kobe Univ., Kobe, Japan; (2) Kobe University, Japan

Pyricularia oryzae, a causal agent of blast disease in various gramineous plants, is composed of subgroups exclusively pathogenic on their original host genera. To clarify genetic mechanisms of the parasitic specialization at the host genus level, we focused on PWT6, one of the avirulence genes involved in the interaction between Eleusine isolate (collected from finger millet) and common wheat. Southern blot analysis using 85 isolates derived from several different subgroups revealed that PWT6 is widely conserved in Eleusine isolates but partially in Oryza isolates. Complementation test revealed that a PWT6 homolog ( $83 \%$ similarity at the amino acid level) derived from an Oryza isolate is also functional against common wheat cv. Chinese Spring (CS). This result suggests that its corresponding resistance gene might be useful for rice breeding for blast resistance. When $\mathrm{F}_{2}$ populations derived from the cross between $\mathrm{CS}$ and cv. Hope (susceptible) were inoculated with a transformant of a Triticum isolate carrying PWT6, resistant and susceptible seedlings segregated in 3:1 ratio suggesting that PWT6 is recognized by a single major gene. This novel gene was designated as Rwt6. Mapping using SSR and CAPS markers revealed that Rwt6 is located on the short arm of $1 \mathrm{D}$ chromosome. The location of Rwt6 is close to the region of Rwt3, a key gene for emergence of the wheat blast fungus.

Adapting to challenges: functions of the Arabidopsis immune adaptor SRFR1 and associated proteins W. GASSMANN (1), B. J. Spears (1), J. Su (1), A. Kimble (1), C. M. Garner (1), M. K. Halane (1), S. H. Kim (2), (1) University of Missouri, Columbia, MO, U.S.A.; (2) Gyeongsang National University, Jinju, Korea, Republic of (South)

Plant immunity needs to be tightly controlled to enable normal plant growth. The balance between immunity and growth occurs on multiple levels and is not fully understood. Using Arabidopsis genetics we identified the adaptor protein SRFR1 that negatively regulates immune signaling triggered by bacterial pathogens. SRFRlencodes a pioneer tetratricopeptide repeat (TPR) protein conserved between plants and animals. The SRFR1 TPR domain has significant sequence similarity to those of transcriptional repressors in yeast and Caenorhabditis elegans. A functional sub-pool of SRFR1 localizes to the nucleus, where it interacts with members of the TCP transcription factor family.TCP transcription factors mainly have been described as regulating developmental processes. We therefore proposed that nuclear SRFR1 functions in a transcriptional repressor complex that balances plant biotic stress resistance and development and will present new data consistent with this model. A second class of SRFR1 interactors consists of immune regulators such as EDS1 and TNL resistance proteins. Following up on the observation that the bacterial effector AvrRps4 directly interacts with EDS1, we discovered that AvrRps4 is a bipartite effector, with both peptides of processed AvrRps4 necessary for triggering resistance. We conclude that SRFR1 and EDS1 may differentially modulate transcriptional regulatory complexes that are targeted by effectors and guarded by resistance proteins. 
Blue light receptor Phototropin 1 increases plant susceptibility to Phytophthora infestans

S. NAQVI (1), Q. He (2), P. Boevink (3), E. M. Gilroy (4), P. R. J. Birch (3), (1) Division of Plant Sciences, University of Dundee, Dundee, U.K.; (2) Division of Plant Science, James Hutton Institute, University of Dundee, Dundee, U.K.; (3) Cell and Molecular Sciences, James Hutton Institute, Dundee, U.K.; (4) The James Hutton Institute, Dundee, U.K.

We have previously shown that $P$. infestans effector PITG_02860 targets host protein StNRL1 to establish infection by degradation of a positive regulator of immunity, StSWAP70. Silencing of StNRL1 significantly reduces infection and accelerates INF1-triggered cell death (ICD) in our model system $N$. benthamiana (He and Naqvi et al 2018 PNAS 115:E7834-E7843). NRL1 belongs to a large family of BTB/POZ domain containing proteins, including two well characterised members in Arabidopsis, NPH3 and RPT2, which play roles in phototropic responses by interacting with blue light receptors Phototropin 1 and 2. Our results indicate that StNRL1 also interacts with Phototropins in planta. We show that Phototropins, similar to StNRL1, suppress the ICD response in $N$. benthamiana, whereas StNPH3 and StRPT2 do not. ICD suppression by photoptropins is compromised by silencing NRL1. Silencing of Phot1 and Phot 2 also seems to reduce P. infestans infection on detached N. benthamiana leaves. Phototropins are activated upon auto-phosphorylation in blue light resulting in the de-phosphorylation and activation of NRL family members, which then can form E3 ligase complexes targeting substrates, including phototropins, for ubiquitination. We have already identified StSWAP70 as a substrate for the StNRL1 E3 ligase complex. Therefore, investigating the role of Phototropin 1 as an upstream regulator of NRL1 is critical to understand the relationship of blue light perception to plant immunity.

The plant immune system constraints bacterial attempts to remain motile while staying undetected Y. BELKHADIR, Gregor Mendel Inst of Molecular Plant Biology GmbH, Vienna, Austria

Plants sense and respond to microbes with an arm of the plant immune system that relies on the detection of microbial-associated molecular patterns (MAMPs) by cell surface pattern-recognition-receptors (PRRs). Stereotypical MAMPs include several epitopes derived from flagellin molecules, the building-block of bacterial flagella. In Arabidopsis, the PRR FLAGELLIN-SENSING 2 (FLS2) detects a highly conserved 22amino acid epitope (flg22) from the $\mathrm{N}$-terminus of flagellin. flg22 is evolutionary conserved and mutations of conserved amino acids that subvert FLS2 detection have deleterious effect on flagellar function and bacterial motility. Here, we set out to define how tradeoffs between motility and host recognition have been molecularly balanced throughout evolution. To circumvent the shortcoming of working in a limited flg 22 natural evolutionary space, we artificially evolved $>1000$ flg22 variants and tested their interaction with FLS2. I will present work defining the molecular constrains and associated mechanisms that impinges on bacterial attempts to evade immune system activities while remaining motile.

Tackling a formidable foe: The re-emergence of wheat stem rust in Western Europe D. G. O. SAUNDERS, John Innes Centre, Norwich, U.K.

Wheat stem rust has been associated with crop failures throughout history. Recent increased incidence in western Europe could signify the return of this formidable foe. Accordingly, alterations in climatic conditions over the past 25 years are consistent with increasingly conducive conditions for pathogen growth and infection. We investigated whether a lack of resistance in modern European wheat varieties, increased presence of the alternate host barberry, alongside changes in climatic conditions could be brewing the perfect storm for stem rust resurgence. In 2013, we recorded wheat stem rust in the U.K. for the first time in over 60 years and showed that over $80 \%$ of wheat varieties were susceptible to this strain. Furthermore, the pathogens alternate host (Berberis spp.) has been increasing in popularity and increased plantings are raising concerns this could facilitate the pathogens re-establishment. In 2016, we documented stem rust for the first time in the U.K. in many decades on its alternate host common barberry. Since, a wider search in 2018 identified stem rust in multiple locations. Furthermore, infection assays showed that aeciospores collected from barberry at many sites are capable of infecting barley, wheat and rye. By integrating spore dispersal models and citizen science to identify barberry locations, we are developing a risk model to better guide re-planting efforts to minimize the risk new plantings could pose to escalating cereal rust diversity in the future.

A comparative population genomics approach to understand virulence in oat crown rust M. FIGUEROA (1), M. E. Miller (2), E. S. Nazareno (1), S. M. Rottschaefer (3), F. Li (1), H. Nguyen-Phuc (1), A. Persoons (4), D. G. O. Saunders (5), C. D. Hirsch (1), E. H. StU.K.enbrock (6), S. F. Kianian (7), (1) University of Minnesota, St. Paul, MN, U.S.A.; (2) Pairwise, Durham, NC, U.S.A.; (3) University of Minnesota, Saint Paul, MN, U.S.A.; (4) John Innes Center, Norwich, U.K.; (5) John Innes Centre, Norwich, U.K.; (6) Max Planck Institute for Evolutionary Biology, Plön, Germany; (7) USDA-ARS Cereal Disease Laboratory, St. Paul, MN, U.S.A.

The fungus, Puccinia coronata f. sp. avenae ( $P c a$ ) causes crown rust and poses a significant challenge to oat production. Genetic resistance is the most sustainable disease management strategy to control crown rust; however, recurrent epidemics demonstrate the vulnerability of this approach as the pathogen evolves to break host resistance. In recent years, $P c a$ populations have changed to display virulence to the vast majority of resistant oat cultivars. To gain insights into the evolution of $P c a$ we took a genomics approach to compare US populations derived from 1990 and 2015 as two distinct years that has captured this drastic shift in virulence. Whole-genome sequencing data was used to determine the genetic differentiation and demographic factors affecting their structure. Given that effector-driven evolution plays an important role in gain-of-virulence, several approaches were used to identify $P c a$ effectors. We identified haustorial effector gene candidates that showed signatures of selection between the 1990 and 2015 populations, as well as presence and absence variation in predicted effector genes. Notably, a genome-wide association study allowed for the identification of the effector candidate $A v r P c 51$. In summary, our research highlights the potential applications of population genomics-based approaches to develop molecular markers for rust virulence and understand plant-microbe co-evolution.

Comparative and population genomic analysis of clonal and sexual populations of Phytophthora infestans

B. J. Knaus (1), J. Tabima (1), S. Shakya (1), H. S. Judelson (2), N. J. GRUNWALD (3), (1) Oregon State University, Corvallis, OR, U.S.A.; (2) University of California, Riverside, CA, U.S.A.; (3) USDA ARS, Corvallis, OR, U.S.A. 
The plant pathogen that caused the Irish potato famine, Phytophthora infestans, continues to reemerge globally. These modern epidemics are caused by clonally reproducing lineages in most of the World. However, a sexual mode of reproduction is observed at its center of origin in Mexico. We conducted a comparative genomic analysis of 47 high coverage genomes to infer changes in genic copy number, ploidy and effector content. We included populations from the center of origin as well as several dominant clonal lineages sampled worldwide. We conclude that sexual populations at the center of origin are diploid as was the lineage that caused the famine, while modern clonal lineages showed increased copy number. Copy number variation was found genome-wide and did not adhere to the two-speed genome hypothesis. Although previously reported, tetraploidy was not found in any of the genomes evaluated. Our work provides novel insights into the emergence of novel clonal lineages and supports a hypothesis whereby higher copy number provides an apparent competitive advantage, while sexual reproduction has reinforced diploidy at the center of origin.

Spatially discrete micro-niches govern root microbiome assembly

B. S. O’BANION, L. O’Neal, G. Alexandre, S. Lebeis, University of Tennessee, Knoxville, TN, U.S.A.

Plant rhizospheres host complex microbial communities that are strongly influenced by exudate composition and microbe-microbe interactions. However, a mechanistic understanding of how chemotaxis towards root metabolites and resulting competitive interactions influence microbiome assembly remains enigmatic. We aim to identify specific microbial factors contributing to plant-microbe and microbe-microbe interactions at distinct spatial locations within a root system. We use a range of biotic complexity (single strain to synthetic community) and spatial heterogeneity (in vitro to in planta) to gain both a mechanistic and holistic understanding of how individual strain phenotypes scale-up to higherorder community dynamics. We first define the fundamental niche of individual bacterial strains in the context of the root by investigating exudate utilization, chemotactic localization, and bulk tissue colonization. We then compare results from in vitro and in planta assays to in silico analyses to classify competitive interactions between all bacterial strains. Lastly, we assess the impact of spatial organization and competition on microbiome establishment using synthetic bacterial communities. We find that individual strains have distinct phenotypes in the context of plant roots and these behaviors can be explained by genetic factors. Additionally, communities with varying levels of competition organize differently along the root and have altered levels of long-term biodiversity.

From high-throughput functional screens to virulence mechanisms of Phytophthora plant pathogens J. Huang, Q. Peng, H. Wu, X. Lu, Y. Wang, S. DONG, Nanjing Agricultural University, China

Irish potato ate blight is a notorious plant disease that threaten global food security. Understanding the disease mechanism is important to design ration disease management. A striking signature of Phytophthora infestans genome is the presence of large effector repertoires, which function to fine-tune host immunity. Functional screening tool are useful to identify effector with virulence functions, but limitations also exist. We previously demonstrated a Phytophthora effector targeting to plant novel splicing factors to reroute global plant pre-mRNA splicing, suggesting manipulation of host mRNA splicing is a pathogen infection strategy. Taking $P$. infestans and tomato interaction as a studying model, we profiled the global tomato mRNA alternative splicing (AS) changes at different time points and experimentally validated a few significant AS events. The mRNA of immune regulatory genes such as transcriptional factors and receptors are subjected to AS changes during infection. We engineered a luciferase reporter system to survey mRNA AS change and made Nicotiana benthamiana transgenic lines. We then screened our Phytophthora effector library, identifying a few Phtophthora effectors that are potentially involved in plant mRNA AS process. The molecular mechanisms of these effector proteins are now under investigations. These researches may provide new insights into microbial manipulation of host plants.

sRNA-mediated interaction between pathogenic fungus Magnaporthe oryzae and cereal model Brachypodium distachyon S. F. ZANINI (1), K. H. Kogel (2), (1) Institute of Phytopathology, Justus-Liebig-Universität Gießen, Giessen, Germany; (2) Institute of Phytopathology, Justus-Liebig-Universität Gießen, Germany

In recent years, fungal pathogens have been found to produce and secrete sRNAs into infected plants in order to silence defence-related host genes, aiding the establishment and maintenance of the disease. Likewise, plants seem to have evolved a similar strategy, hampering the infection by targeting via sRNAs either virulence or essential genes of microbial pathogens, a phenomenon called bidirectional or cross-kingdom RNAi (ck-RNAi). Given the small number of examples of ck-RNAi in literature, further studies are required to assess how far this RNA-mediated mechanism is evolutionary conserved. With the aim of detecting such RNA-based communication in a new system, we assessed the accumulation of sRNAs during the interaction of Magnaporthe oryzae (Mo) and Brachypodium distachyon (Bd). By concomitant sequencing of sRNA and mRNA fractions from infected leaves of the biotrophic and necrotrophic infection phases, infected roots, Mo axenic culture and non-infected Bd samples we identified Bd and Mo sRNAs higher expressed in the different phases and tissues. Additionally, for selected ck-sRNA candidates we observed significant downregulation of predicted mRNA targets in the interacting organism. Overall, we found significant differences in sRNA accumulation in roots and leaves as well as in the biotrophic and necrotrophic phases, suggesting that ck-RNAi is not only specific to the interacting organisms, but also to the tissue where the interaction occurs and the pathogen lifestyle.

Plant Interactions with Pathogenic and Commensal Bacteria

T. Nobori, Y. Cao, Y. Wang, F. Entila, K. TSUDA, Max Planck Institute for Plant Breeding Research, Cologne, Germany

Despite extensive studies into the molecular and genetic bases of plant immunity, we still lack a fundamental knowledge of how plant immunity affects pathogen metabolism to control their growth in plants. We studied bacterial transcriptome responses of the foliar pathogen Pseudomonas syringae in leaves of Arabidopsis thaliana and identified specific immune-responsive bacterial genes and processes, including those that are activated in susceptible plants and suppressed by plant immunity. Expression patterns of immune-responsive bacterial genes at an early time point explained later bacterial growth levels in different host genotypes, indicating that bacterial transcriptome response is a critical determinant for the interaction. A large scale in planta bacterial transcriptome analysis also revealed previously undescribed gene regulatory logic in bacteria. We also determined in planta bacterial proteome responses and found that specific bacterial proteins were suppressed or degraded by plant immunity. These results illuminate impacts of host immunity on bacterial metabolisms and provide clues for how plants control bacterial behavior and 
growth. We extended our analysis to members of the plant microbiota to understand the commonality and specificity of plant immunity on different bacterial species and microbiota assembly and function regulated by host immunity.

Convergent gain and loss of genomic islands drive lifestyle changes in plant-associated Pseudomonas

R. A. MELNYK (1), S. S. Hossain (2), C. H. Haney (3), (1) The University of California Davis, U.S.A.; (2) University of Toronto, Toronto, ON, Canada; (3) The University of British Columbia, Vancouver, BC, Canada

Host-associated bacteria can have both beneficial and detrimental effects on host health. While some of the molecular mechanisms that determine these outcomes are known, little is known about the evolutionary histories of pathogenic or mutualistic lifestyles. Using the model plant Arabidopsis, we found that closely related strains within the Pseudomonas fluorescens species complex promote plant growth and occasionally cause disease. To elucidate the genetic basis of the transition between commensalism and pathogenesis, we developed a computational pipeline and identified genomic islands that correlate with outcomes for plant health. One island containing genes for lipopeptide biosynthesis and quorum sensing is required for pathogenesis. Conservation of the quorum sensing machinery in this island allows pathogenic strains to eavesdrop on quorum signals in the environment and coordinate pathogenic behavior. We found that genomic loci associated with both pathogenic and commensal lifestyles were convergently gained and lost in multiple lineages through homologous recombination, possibly constituting an early step in the differentiation of pathogenic and commensal lifestyles. Collectively this work provides novel insights into the evolution of commensal and pathogenic lifestyles within a single clade of host-associated bacteria.

Pathogen-induced acidification across the plasma membrane regulate the growth-defense balance of plants C. SANCHEZ-RODRIGUEZ, ETH Zürich; D-BIOL; IMPB, Switzerland

Environmental adaptation of organisms relies on the fast perception and response to external signals, which leads to developmental changes. Plant cell growth is strongly dependent on CW remodeling. However, little is known about CW-related sensing of biotic stimuli and the downstream mechanisms that coordinate growth and immune responses. We anticipated a short- and long-termed CW reorganization in response to microbes. To test this hypothesis, we use the model pathosystem Arabidopsis- Fusarium oxysporum5176 (Fo5176). Using high-resolution livecell imaging, we observed a pH-dependent alteration of the cellulose synthesis machinery and root elongation within five minutes of hypha contact. We generated genetically encoded $\mathrm{pH}$ sensors to determine absolute $\mathrm{pH}$ changes across the plasma membrane in response to the vascular wilt fungus. A rapid apoplast acidification by phosphorylation-based proton pump activation was followed by an acidification of the cortical side of the plasma membrane in response to Fo5176. By developing a novel plant infection assay, we confirmed a direct influence of the proton chemical gradient modulation on the pathogenicity of the fungus. All these effects were dependent on the COMPANION OF CELLULOSE SYNTHASE proteins that are thus at the nexus of plant growth and defence. Our discoveries show a remarkable connection between plant biomass production, immunity, and $\mathrm{pH}$ control, and advances our ability to investigate the plant growth-defence balance.

Close proximity of the TIR domain of diverse plant intracellular immune receptor drives signaling activation from different cell compartments

M. BERNOUX (1,2), X. Zhang (3), J. Chen (3), J. Hu (4), P. N. Dodds (5), (1) INRA/CNRS, Toulouse, France; (2) CSIRO Agriculture, Canberra, Australia; (3) CSIRO, Canberra, Australia; (4) China Agricultural University, Beijing, China; (5) CSIRO Agriculture and Food, Canberra, ACT, Australia

Members of the NOD-like receptor family play a central role in plant and animal immunity. In plants, they can specifically recognise pathogen effectors to activate a strong and rapid immune response. However, early steps that connect activated NLRs to immune signaling induction is still very obscure. In animals, NLR activation is followed by assembly of high-order complexes, bringing the N-terminal domains into close proximity, and allowing the recruitment of signaling partners. We previously showed that self-association of the N-terminal TIR domain of several plant NLRs is required for their signaling activity, but the steps following TIR self-association leading to signaling induction is unknown. Given that NLRs localize to various subcellular compartments, we investigated where NLRs initiate signaling following TIR self-association. Here, we used isolated TIR domains from NLRs that show different subcellular localization, flax L6 (Golgi membrane localised), and the Arabidopsis RPS4/RRS1 and SNC1 (nucleocytosolic). By forcing isolated TIR dimerization, we showed that close proximity of plant NLR TIRs is essential to activate TIR-mediated immune signaling, similarly to the activation mechanism of animal NLRs. While this seems to be conserved between L6, RPS4 and SNC1, we showed by using mis-localised TIR variants, that L6 TIR signals from the cytosol whereas RPS4 and SNC1 TIR can signal from both the nucleus and the cytosol.

Bacterial medium chain 3-hydroxy fatty acid metabolites trigger LORE-mediated immunity in Arabidopsis thaliana

A. Kutschera (1), C. Dawid (1), N. Gisch (2), C. Schmid (1), L. Raasch (1), T. Gerster (1), M. Schäffer (1), E. Smakowska (3), Y. Belkhadir (3), C. Vlot (4), C. E. Chandler (5), R. Schellenberger (6), D. Schwudke (2), R. K. Ernst (5), S. Dorey (6), R. Hueckelhoven (1), T. Hofmann (1), S. RANF (1), (1) Technical University of Munich, Freising, Germany; (2) Research Center Borstel, Borstel, Germany; (3) Gregor Mendel Institute of Molecular Plant Biology (GMI), Vienna, Austria; (4) Institute of Biochemical Plant Pathology, Helmholtz Zentrum Muenchen, Germany; (5) University of Maryland School of Dentistry, Department of Microbial Pathogenesis, Baltimore, U.S.A.; (6) Reims Champagne-Ardenne University, Reims, France

In plants, recognition of microbe-associated molecular patterns (MAMPs) by specific cell-surface immune receptors activates pattern-triggered immunity. Lipopolysaccharide (LPS), particularly the lipid A moiety, of Gram-negative bacteria is sensed as MAMP in mammals through different receptors. Lipid A is also considered a MAMP in plants. We previously showed that the bulb-type lectin S-domain receptor-like kinase LORE (LipoOligosaccharide-specific Reduced Elicitation) mediates plant immune responses to Pseudomonas and Xanthomonas, but not enterobacterial, lipid A/LPS preparations in Arabidopsis thaliana (1). Here, we demonstrate that LORE senses synthetic and bacterial medium chain 3-hydroxy fatty acid (mc-3-OH-FA) metabolites to elicit PTI. Mc-3-OH-FAs are sensed in a chain length-specific manner with free ( $R$ )-3hydroxydecanoic acid representing the strongest immune elicitor. We found that elicitor-active mc-3-OH-FAs co-purify with LPS/lipid A. Re- 
purification of LPS depletes mc-3-OH-FAs. Such mc-3-OH-FA-depleted LPS preparations do not trigger LORE-dependent PTI. Likewise, other bacterial compounds comprising mc-3-OH-acyl building blocks but being devoid of free mc-3-OH-FAs, such as rhamnolipids, lipopeptides, and acyl-homoserine-lactones, also do not trigger LORE-dependent PTI. Taken together, we show that plants sense low-complexity bacterial metabolites to trigger immune responses.

The SCAR/WAVE complex protein API controls the establishment of host cell wall properties essential for microbial infection. A. GAVRIN, Cambridge University, Cambridge, U.K.

The cell wall is a primary interface between plant cells and their immediate environment and thus must balance multiple functionalities including development, structural support, entry of beneficial microbes, and protection against pathogens. To identify genes commonly required for the accommodation of pathogenic and symbiotic microbes, we carried out root oomycete infection assays on Medicago truncatula mutants affected in rhizobia-root nodule/arbuscular mycorrhiza symbiosis development. We identified and characterised the Medicago gene API, as a susceptibility factor contributing to the microbial infection. API encodes a SCAR2 protein, a subunit of the SCAR/WAVE actin regulatory complex. We demonstrate that API controls establishment of cell wall properties required for efficient root infection by the pathogen Phytophthora palmivora.Resistance was caused by modification of the cell wall architecture. We demonstrated that altered endomembrane trafficking dynamics in api mutants results in the distortion of secreted cell wall remodelling factors. This leads to changes in biochemical and rheological properties of root cell walls that impair pathogen root entry without affecting plant development. Our work demonstrates that alteration in cell wall architecture at a specific stage of root development contributes to disease resistance during compatible interactions without compromising root growth, offering a potential route to quantitative root resistance against Phytophthora.

Subversion of plant immunity at the host-pathogen interface T. O. BOZKURT (1), P. Pandey (1), Y. Tumtas (1), C. Duggan (1), A. Y. Leary (1), Z. Savage (1), A. Toufexi (1), M. P. Contreras (2), M. E. Segretin (3), Y. F. Dagdas (4), S. Kamoun (2), (1) Imperial College London, U.K.; (2) The Sainsbury Laboratory, University of East Anglia, Norwich Research Park, Norwich, U.K.; (3) INGEBI-CONICET, Buenos Aires, Argentina; (4) Gregor Mendel Institute of Molecular Plant Biology, Vienna, Austria

During plant invasion, the Irish potato famine pathogen Phytophthora infestans penetrates host cells through hyphal extensions known as haustoria. Haustoria are enveloped by a host-derived membrane known as the extra-haustorial membrane (EHM) whose functions and biogenesis are poorly understood. Through this interface, the pathogen secretes effector proteins which comprehensively subvert host immune responses. An interesting group of effectors particularly target the EHM, some of which interfere with defense-related secretory pathways. Using the perihaustorial effectors as molecular probes, we identfied a series of plant defense components deployed to the EHM as well as pathogen strategies that antagonize plant focal immunity. These processes include; (i) modulation of plant autophagy at the haustorial interface; (ii) trafficking of immune receptors towards the EHM; (iii) recruitment of chloroplasts to the pathogen interface. I will discuss our recent discoveries on these processes, focusing particularly on the reprograming of defense-related autophagy and immune recognition at the pathogen interface. The emerging paradigm is that perihaustorial effectors counteract focal immune responses and remodel the host-microbe interface for safe and efficient nutrient absorption. A better understanding of the cellular trafficking pathways and the signaling events at various plant-pathogen interfaces would enable novel strategies to tackle filamentous plant pathogens.

Investigating host specificity and the role of core effector proteases for Clavibacter infection of tomato S. P. Thapa (1), Q. Lyu (1), D. M. Stevens (1), M. L. O’Leary (1), M. Agnes-Jacques (2), B. Gilbertson (1), G. L. COAKER (1), (1) University of California, Davis, CA, U.S.A.; (2) INRA, Université d'Angers, Beaucouzé, France

Species within the Clavibacter genus are Gram-positive bacteria that colonize plant xylem vessels causing significant economic losses. Each species of plant pathogenic Clavibacter primarily infects one main host, with Clavibacter michiganensis $(\mathrm{Cm})$ causing bacterial wilt and canker of tomato. The molecular mechanisms required for infection of plants by Gram-positive bacteria are poorly understood relative to their Gramnegative counterparts. Phylogenetic analyses using 1,258 orthologous genes were used to resolve relationships among 37 Clavibacter species isolated from different hosts. Pathogenic $\mathrm{Cm}$ isolated from tomato formed a strongly supported monophyletic group. In contrast, endophytic Clavibacter strains from tomato clustered with strains causing disease in other plants and were capable of causing disease on those plants. We performed comparative genomic analyses to identify conserved secreted type II effector proteins. Core secreted effectors in $\mathrm{Cm}$ are located in a large pathogenicity island and comprise three families of serine proteases. This pathogenicity island is likely important for tomato host specificity, as it is conserved in $\mathrm{Cm}$, but absent in other pathogenic Clavibacter species. The abundance, enzymatic activity and importance of specific serine proteases for pathogenicity on tomato will be reported.

Spatially distinct sugar signatures along intracellular interfaces of filamentous microbes S. SCHORNACK (1), T. Torode (2), (1) Sainsbury Laboratory, University of Cambridge, Cambridge, U.K.; (2) Cambridge University, Sainsbury Laboratory (SLCU), Cambridge, U.K.

Haustoria of pathogenic fungi and oomycetes and arbuscules of symbiotic arbuscular mycorrhiza (AM) fungi are structures formed within plant cells during biotrophy. While a surrounding cellulosic plant cell wall is missing, many cell wall components are still secreted across a specialised membrane into the space between both organisms. Interface-targeted plant proteins have helped defining functionally different domains such as arbuscule branch and trunk domains. Their localisation relies on a repolarisation of secretory processes coupled with their expression. Given such temporal/spatial relationship of arbuscule secretion we hypothesized that the periarbuscular space of branch and trunk domains will markedly differ in its composition of secreted cell wall components. Using monoclonal antibodies towards cell wall components we identified specific glycan-modifications that have spatially distinct or equal distribution along the arbuscule. Comparing these signatures in arbuscules formed in Nicotiana benthamiana, Medicago truncatula and liverworts allowed us to identify host- and colonisation-type associated differences. We are now extending this work into intracellular haustoria of pathogenic filamentous microbes to define common and specific signatures of matrix 
carbohydrates at the interface between plants and microbes and will therefore further our understanding on how similar pathogenic and symbiotic interfaces are.

A bacterially produced polyamine induces plant systemic susceptibility to pathogens

R. A. Melnyk (1), P. Beskrovnaya (2), Z. Liu (2), Y. Song (2), C. H. HANEY (2), (1) The University of California Davis, U.S.A.; (2) The University of British Columbia, Vancouver, BC, Canada

Plant root-associated microbes promote plant growth, in part by the induction of systemic resistance (ISR) to foliar pathogens. In an attempt to find novel growth-promoting and ISR inducing strains, we previously identified strains of root-associated Pseudomonas spp. that promote plant growth but unexpectedly induced systemic susceptibility (ISS) to foliar pathogens. Here we demonstrate that the ISS-inducing phenotype is common among root-associated Pseudomonas spp. and we identified the underlying genetic and molecular basis of ISS. Using comparative genomics we identified a single $P$. fluorescens locus containing a novel periplasmic predicted spermidine biosynthesis gene speE2 that is unique to ISS strains. We generated a clean deletion of the speE2 gene in two ISS strains and found that speE2 is necessary for the ISS phenotype. Spermidine but not spermine is sufficient to phenocopy ISS strains. The ISS locus is present in diverse bacteria and has previously been implicated in pathogenesis in animals. Collectively these data show that a single bacterially derived molecule can modulate systemic plant immunity.

Isolation, characterization, and migrational patterns of bacterial endophytes during germination of S. plicata (Orchidaceae) A. Badran, T. Seitz, D. Hunter, T. Lorenz, S. DARLING-NOVAK, University of La Verne, La Verne, CA, U.S.A.

Many bacterial endophytes are seed borne, yet how they populate germinating seedlings has not been well established. In this work, an orchid system, Spathoglottis plicata, was studied to address the question of microbial migration and to characterize bacterial endophytes obtained from a relatively vulnerable, underdeveloped seed. Orchids produce reserve-deficient, dust-sized seeds that are released from their pod in globular-like stage of embryogenesis, making them an ideal candidate for microbial assistance with regard to stress and/or development. Cultivation-dependent and independent techniques were conducted on the endophytic bacterial species in seed and seedlings grown under gnotobiotic conditions. The seed harbored 153 species of bacteria, while seedling root and shoot housed 112 and 91, respectively, with Bradyrhizobium elkanii being most abundant in the root, and Acinetobacter johnsonii most predominant in the shoot. Of the ten species isolated from protocorm homogenate, most were Bacillus. To evaluate bacterial migration, motile bacteria were labeled with an anti-flagellin antibody throughout germination. Endophytes were associated with the seed coat, and at later stages of germination, aggregated within the coat and protocorm. Ultimately, the endophytes localized at the stomata and protocorm hairs. These findings are suggestive of co-aggregate activity, a characteristic of the predominate species identified in the leaf. 